\title{
Robust small area estimation under spatial non-stationarity
}

\author{
Claudia Baldermann*, Nicola Salvati**, and Timo Schmid* \\ *Institute of Statistics and Econometrics, Freie Universität Berlin, Berlin, Germany \\ ${ }^{* *}$ Economic Department, University of Pisa, Pisa, Italy
}

\begin{abstract}
Geographically weighted small area methods have been studied in literature for small area estimation. Although these approaches are useful for the estimation of small area means efficiently under strict parametric assumptions, they can be very sensitive to outliers in the data. In this paper, we propose a robust extension of the geographically weighted empirical best linear unbiased predictor (GWEBLUP). In particular, we introduce robust projective and predictive small area estimators under spatial non-stationarity. Mean squared error estimation is performed by two different analytic approaches that account for the spatial structure in the data. The results from the model-based simulations indicate that the proposed approach may lead to gains in terms of efficiency. Finally, the methodology is demonstrated in an illustrative application for estimating the average total cash costs for farms in Australia.
\end{abstract}

Keywords: Bias correction, geographical weighted regression, mean squared error, model-based simulation, spatial statistics

\section{Introduction}

The demand for reliable statistics on regionally disaggregated levels has been grown in the last decades. Sample surveys provide a cost efficient way to obtain estimates for the quantity of interest mainly on higher geographic levels (like countries or regions). In most practical situations, the sample sizes on lower geographic levels (like counties or municipalities) are not large enough to provide precise direct estimates. These levels are called small areas. Several model-based approaches for producing small area estimates have been proposed in literature to tackle this problem. We refer to Rao (2003), Jiang and Lahiri (2006) and Rao and Molina (2015) for a general introduction and an overview.

The production of reliable small area estimates is based on the availability of accurate auxiliary information. In many situations, additional spatial information such as coordinates and distances between small areas is available and can be incorporated in the model. Several authors (Longford, 2010; Chandra et al., 2012; D'Alo et al., 2012; Porter et al., 2014) have already demonstrated in literature that borrowing strength over space offers some gains in terms of efficiency in small area estimation. One approach for 
incorporating the spatial correlation in the data is to allow for spatially correlated random effects by using conditional or simultaneous autoregressive processes, while the regression coefficients are spatially invariant (Rao, 2003; Singh et al., 2005; Pratesi and Salvati, 2008). An alternative approach is to incorporate the spatial structure in the data into a nonparametric spatial spline model for small area estimation (Opsomer et al., 2008; Giusti et al., 2012). The spatial correlation is captured by spatially varying auxiliary information whereas the regression coefficients are spatially invariant. A third approach assumes that the regression coefficients vary spatially across the small area of interest. This situation is referred to as spatial non-stationarity (Brunsdon et al., 1996; Fotheringham et al., 2002). Recently, Chandra et al. (2012) and Salvati et al. (2012) discussed small area estimation for unit-level models for this situation. In particular, Chandra et al. (2012) proposed a geographical weighted empirical best linear unbiased predictor (GWEBLUP) for small area estimation under spatial non-stationarity. Chandra et al. (2015) presented a spatially non-stationary Fay-Herriot model (Fay and Herriot, 1979). From a Bayesian perspective, Sugasawa et al. (2015) introduced an empirical Bayes method with spatially non-stationary hyperparameters for area-level data.

Although these approaches for small area estimation under spatial correlation are efficient under strict parametric assumptions, they can be strongly influenced by departures from the underlying distributions or by outliers. Outliers are correctly recorded observations which are extremely different compared to the whole sample (Chambers, 1986). Sinha and Rao (2009) used influence functions for estimating robust parameters in a linear mixed model and propose a robust empirical best linear unbiased predictor (REBLUP) for small area estimation. An alternative approach to outlier robust small area estimation based on M-quantile methods is introduced in Chambers and Tzavidis (2006). Both approaches normally lead to biased small area estimators with smaller variances because they assume that all non-sampled values follow exactly the assumed robust working model. Chambers et al. (2014) denote such approaches as robust projective. Dongmo-Jiongo et al. (2013) and Chambers et al. (2014) corrected for the bias of the robust small area estimators by adding an additional correction term. Such bias-corrected robust estimators are denoted as robust predictive because they try to predict the contribution of the outliers in the population (Chambers et al., 2014). Recently, Schmid and Münnich (2014) and Schmid et al. (2016) discussed the robust projective and predictive estimators under spatially correlated random effects.

This paper is, to the best of our knowledge, the first attempt to propose a robust approach to small area estimation for spatial non-stationarity data. In order to account for potential outliers and spatially varying regression coefficients in the data we control the impact of outliers on the geographically weighted regression by an influence function; i.e. lower weights are given to the outliers in the fitting process.

The structure of the paper is as follows. In Section 2 we give a short review regarding the GWEBLUP and present the proposed robust extensions. In particular, we introduce robust projective and predictive small area estimators under spatial non-stationarity. Section 3 presents two analytic approaches for the mean squared error (MSE) estimation of the proposed estimators that account for the spatial structure in the data. The first method is based on a pseudo-linearization introduced by Chambers et al. (2011), whereas the second approach extends the full-linearization method of Chambers et al. (2014) to spatial 
non-stationarity. In Section 4 the performance of the proposed methodology is empirically assessed by a model-based simulation study under different scenarios. The introduced methods are applied to the Agricultural and Grazing Industries Survey from Australia in Section 5. Finally, in Section 6 we discuss the main results of the paper and provide some avenues for further research.

\section{Robust geographically weighted EBLUP}

This section provides an overview regarding small area methods under spatial non-stationarity. In particular, we will review the geographically weighted EBLUP of Chandra et al. (2012) in Subsection 2.1. Afterwards, we introduce robust projective and robust predictive extensions of the GWEBLUP in Subsection 2.2 and 2.3 respectively.

\subsection{Geographically weighted EBLUP}

We assume that the target population $U$ of size $N$ is divided into $M$ disjoint small areas and that unitlevel data is available. The population units are denoted by $j$ and the small areas by $i$. Each small area $i$ contains a known number $N_{i}$ of units. The vector $\boldsymbol{y}$ stands for the target variable in the population $U$ whereas $y_{i j}$ represents the realization of $y$ for unit $j\left(j=1, \ldots, N_{i}\right)$ in small area $i(i=1, \ldots, M)$. The matrix $\boldsymbol{X}$ contains $p$ unit-level auxiliary variables where the values $\mathbf{x}_{i j}$ of $p \times 1$ vector denotes the individual level covariates for unit $j$ in small area $i$. Furthermore, the matrix $\boldsymbol{Z}$ indicates area level covariates where the $\boldsymbol{z}_{i j}$ denotes the value of $\boldsymbol{Z}$ for unit $j$ in area $i$. We assume that the covariates $\mathbf{x}_{i j}$ and $\mathbf{z}_{i j}$ are linearly related to $y_{i j}$ and that all small area averages of $\mathbf{x}_{i j}$ and $\mathbf{z}_{i j}$ are known. The sample $s$ of size $n$ is drawn from the population by using a non-informative sampling design given $\mathbf{x}_{i j}$ and $\mathbf{z}_{i j}$. The subscripts $s$ and $r$ stand for the $n$ sampled and the $N-n$ non-sampled quantities from the population respectively. We use the index $i$ to denote the restriction to the specific area $i$.

The aim is to use the sample $s$ and additional covariates $\mathbf{x}_{i j}$ and $\mathbf{z}_{i j}$ to predict the small area averages of the target variable $y$. Battese et al. (1988) introduced the standard approach to model-based small area estimation with unit-level data. The Battese, Harter and Fuller model is a linear mixed model (LMM) with area-specific random effects:

$$
\boldsymbol{y}=\boldsymbol{X} \boldsymbol{\beta}+\boldsymbol{Z} \boldsymbol{v}+\boldsymbol{e}
$$

where $\boldsymbol{\beta}$ stands for the $(p \times 1)$ vector of regression coefficients, $\boldsymbol{v} \sim N\left(0, \boldsymbol{\Sigma}_{v}\right)$ denotes the $(M \times 1)$ vector of area specific random effects and $\boldsymbol{e} \sim N\left(0, \boldsymbol{\Sigma}_{e}=\sigma_{e}^{2} \boldsymbol{I}_{N}\right)$ is the $(N \times 1)$ vector of individual errors. $\boldsymbol{I}_{N}$ represents the identity matrix of dimension $N$. The covariance matrix $\boldsymbol{\Sigma}_{v}$ simplifies under to assumption of independent random effects to $\boldsymbol{\Sigma}_{v}=\sigma_{v}^{2} \boldsymbol{I}_{M}$. As the random effects $\boldsymbol{v}$ and the individual errors $\boldsymbol{e}$ are independent, the covariance matrix of $\boldsymbol{y}$ is defined by $\boldsymbol{V}=\boldsymbol{\Sigma}_{e}+\boldsymbol{Z} \boldsymbol{\Sigma}_{v} \boldsymbol{Z}^{T}$. The empirical best linear unbiased predictor (EBLUP) for the small area mean $\bar{y}_{i}$ is then

$$
\hat{\bar{y}}_{i}^{E B L U P}=N_{i}^{-1}\left\{\sum_{j \in s_{i}} y_{i j}+\sum_{j \in r_{i}} \hat{y}_{i j}\right\}=N_{i}^{-1}\left\{\sum_{j \in s_{i}} y_{i j}+\sum_{j \in r_{i}}\left(\boldsymbol{x}_{i j}^{T} \hat{\boldsymbol{\beta}}+v_{i}\right)\right\},
$$


where $\hat{\boldsymbol{\beta}}=\hat{\boldsymbol{\beta}}(\hat{\boldsymbol{\theta}})$ denotes the empirical best linear unbiased estimator and $\hat{\boldsymbol{v}}(\hat{\boldsymbol{\theta}})$ stands for the empirical best linear unbiased predictor (Henderson, 1975). $\hat{\boldsymbol{\theta}}$ is defined by the maximum likelihood or the restricted maximum likelihood estimator of $\boldsymbol{\theta}=\left(\sigma_{v}^{2}, \sigma_{e}^{2}\right)$ (Harville, 1977). We refer to Rao (2003), Jiang and Lahiri (2006) and Rao and Molina (2015) for a comprehensive review of small area estimation based on these models.

The EBLUP (2) is optimal in terms of efficiency under the assumptions of the LMM (1). In particular, the regression coefficients $\boldsymbol{\beta}$ are spatially invariant (spatial stationary) in the LMM, i.e. the relationship between $y_{i j}$ and $\mathbf{x}_{i j}$ is the same in the target area. Recent literature has demonstrated that there are realistic situations where the linear relationship is not constant (Opsomer et al., 2008; Chandra et al., 2012; Salvati et al., 2012; Chambers et al., 2016).

Following Brunsdon et al. (1996), one way to model this problem is to allow for local linear (spatial non-stationary) models for the target variable $y$ by geographically weighted regression (GWR). Chandra et al. (2012) extended these ideas to the context of small area estimation. The local LMM with respect to an arbitrary spatial location $u_{k}$ is given by

$$
\boldsymbol{y}=\boldsymbol{X} \boldsymbol{\beta}\left(u_{k}\right)+\boldsymbol{Z} \boldsymbol{v}\left(u_{k}\right)+\boldsymbol{W}_{k}^{-1 / 2} \boldsymbol{e}
$$

where $\boldsymbol{\beta}\left(u_{k}\right)$ stands for the $(p \times 1)$ vector of regression coefficients specific to the location $u_{k}$ and $\boldsymbol{W}_{k}=\operatorname{diag}_{l \in s}\left(w_{l k}\right)$. The element $w_{l k}=w\left(u_{k}, u_{l}\right)$ (for all $l \in s$ ) is a weighting function that decreases as the distance between the locations $u_{k}$ and $u_{l}$ increases. Chandra et al. (2012) used an Euclidean weighting function between the locations $u_{k}$ and $u_{l}$. A comprehensive review of different weighting functions in GWR is provided in Fotheringham et al. (2002).

The geographically weighted EBLUP (GWEBLUP) for the mean $\bar{y}_{i}$ in area $i$ under the spatial nonstationary model (3) is then

$$
\hat{\bar{y}}_{i}^{G W E B L U P}=N_{i}^{-1}\left\{\sum_{j \in s_{i}} y_{i j}+\sum_{j \in r_{i}} \hat{y}_{i j}^{g w}\right\}=N_{i}^{-1}\left\{\sum_{j \in s_{i}} y_{i j}+\sum_{j \in r_{i}}\left(\boldsymbol{x}_{i j}^{T} \hat{\boldsymbol{\beta}}_{\left(u_{i j}\right)}^{g w}+\hat{v}_{i\left(u_{i j}\right)}^{g w}\right)\right\},
$$

where the superscript $g w$ indicates that the parameters depend on geographically weighted regression. $\hat{\boldsymbol{\beta}}_{\left(u_{k}\right)}^{g w}$ denotes the geographically weighted empirical BLUE (GWEBLUE) of $\boldsymbol{\beta}_{\left(u_{k}\right)}^{g w}$ and $\hat{\boldsymbol{v}}_{\left(u_{k}\right)}^{g w}$ stands for geographically weighted EBLUP (GWEBLUP) of the vector of random effects at $u_{k}$ (Chandra et al., 2012). Details regarding the algorithms for the estimating the model parameters are provided in Chandra et al. (2012). It is important to notice that for the GWEBLUP (4) all parameters have to be estimated for each sampled and non-sampled unit in the population what makes the estimation computationally demanding.

\subsection{Robust extension of the GWEBLUP}

The EBLUP (2) and the GWEBLUP (4) are optimal when the corresponding model assumptions hold. In particular, both estimators assume normality for the random effects $\boldsymbol{v}$ and for the error terms $e$. However, the presence of outliers can violate these assumptions. The influence of contamination on the estimation 
can be reduced by substituting $\hat{\boldsymbol{\beta}}$ and $\hat{\boldsymbol{v}}$ by robust estimators. Sinha and Rao (2009) applied an influence function $\psi$ on the residuals in the maximum likelihood (ML) estimating equations and obtained robust estimates $\hat{\boldsymbol{\beta}}^{\psi}$ and $\hat{\boldsymbol{v}}^{\psi}$ by an iterative algorithm. Using these robust alternatives Sinha and Rao (2009) proposed an outlier robust version of the EBLUP (REBLUP) for $\bar{y}_{i}$ in area $i$ by

$$
\hat{\bar{y}}_{i}^{R E B L U P}=N_{i}^{-1}\left\{\sum_{j \in s_{i}} y_{i j}+\sum_{j \in r_{i}}\left(\boldsymbol{x}_{i j}^{T} \hat{\boldsymbol{\beta}}^{\psi}+\hat{v}_{i}^{\psi}\right)\right\} .
$$

In the following we propose an outlier robust version of the GWEBLUP (RWGEBLUP). Similar to Sinha and Rao (2009) we first maximise the density of $\boldsymbol{y}$ with respect to $\boldsymbol{\beta}\left(u_{k}\right)$ and $\boldsymbol{\theta}$ by solving the ML estimating equations. Then, the robust estimates are used for the estimation of the random effects $\boldsymbol{v}\left(u_{k}\right)$ (Fellner, 1986).

Given the response vector $\boldsymbol{y}$, the robustified ML estimating equations with respect to $\boldsymbol{\beta}\left(u_{k}\right)$ and $\boldsymbol{\theta}\left(u_{k}\right)$ under the local LMM (3) have the form

$$
\begin{aligned}
\alpha\left(\boldsymbol{\beta}\left(u_{k}\right)\right) & =\boldsymbol{X}^{T} \boldsymbol{V}_{k}^{-1} \boldsymbol{U}_{k}^{1 / 2} \psi\left(\boldsymbol{r}_{k}\right)=0 \\
\Phi\left(\theta_{l}\left(u_{k}\right)\right) & =-\operatorname{tr}\left(\boldsymbol{V}_{k}^{-1} \frac{\partial \boldsymbol{V}_{k}}{\partial \theta_{l}} \boldsymbol{K}\right)+\boldsymbol{U}_{k}^{1 / 2} \psi\left(\boldsymbol{r}_{k}\right) \boldsymbol{V}_{k}^{-1} \frac{\partial \boldsymbol{V}_{k}}{\partial \theta_{l}} \boldsymbol{V}_{k}^{-1} \boldsymbol{U}_{k}^{1 / 2} \psi\left(\boldsymbol{r}_{k}\right)^{T}=0
\end{aligned}
$$

where $\boldsymbol{r}_{k}=\boldsymbol{U}_{k}^{-\frac{1}{2}}\left(\boldsymbol{y}-\boldsymbol{X} \boldsymbol{\beta}_{\left(u_{k}\right)}\right), \boldsymbol{U}_{k}=\operatorname{diag}\left(\boldsymbol{V}_{k}\right)$ and $\boldsymbol{V}_{k}=\boldsymbol{Z} \boldsymbol{\Sigma}_{v} \boldsymbol{Z}^{T}+\boldsymbol{\Sigma}_{e} \boldsymbol{W}_{k}^{-1} . \boldsymbol{K}$ is a diagonal matrix with $\boldsymbol{K}=E\left(\psi_{b}^{2}(a)\right) \boldsymbol{I}_{n}$ where $a$ is standard normally distributed and $\boldsymbol{I}_{n}$ denotes the identity matrix of size $n$. We use the Huber influence function $\psi(a)=a \min (1, c /|a|)$ with a fixed tuning constant $c=1.345$ (Huber, 1964). Note that the robust ML equations (6) and (7) have to be solved for each individual in the population what makes the estimation computationally demanding. Following Chandra et al. (2012), we apply a quasi-maximum likelihood approach which restricts the variance components $\boldsymbol{\theta}$ to be constant at every sample location. The robust ML equation for the variance components $\boldsymbol{\theta}$ reduces to

$$
\Phi\left(\theta_{l}\right)=-\operatorname{tr}\left(\boldsymbol{V}^{-1} \frac{\partial \boldsymbol{V}}{\partial \theta_{l}} \boldsymbol{K}\right)+\boldsymbol{U}^{1 / 2} \psi(\boldsymbol{r}) \boldsymbol{V}^{-1} \frac{\partial \boldsymbol{V}}{\partial \theta_{l}} \boldsymbol{V}^{-1} \boldsymbol{U}^{1 / 2} \psi(\boldsymbol{r})^{T}=0
$$

where $\boldsymbol{r}=\boldsymbol{U}^{-\frac{1}{2}}\left(\boldsymbol{y}-\boldsymbol{\rho}_{s}\right)$. The projection $\boldsymbol{\rho}_{s}$ is given by $\boldsymbol{\rho}_{s}=\left(\boldsymbol{X} \circ \boldsymbol{B}_{s}\right) \mathbf{1}_{p}$ where $\mathbf{1}_{p}$ is a $p$-vector of ones and $\circ$ denotes the element wise product of matrices, also known as the Hadamard product of matrices. $\boldsymbol{B}_{s}$ and $\boldsymbol{B}_{r}$ denote the matrices of the regression coefficients $\boldsymbol{\beta}\left(u_{k}\right)$ for the sampled and non-sampled units respectively and $\boldsymbol{B}=\left(\boldsymbol{B}_{s}, \boldsymbol{B}_{r}\right)$. Using the robust estimates from (6) and (8), we estimate the robust random effects by solving the equation proposed by Fellner (1986)

$$
\Delta(\boldsymbol{v})=\boldsymbol{Z}^{T} \boldsymbol{\Sigma}_{e}^{-1 / 2} \psi\left(\boldsymbol{\Sigma}_{e}^{-1 / 2}\left(\boldsymbol{y}-\boldsymbol{\rho}_{s}-\boldsymbol{Z} \boldsymbol{v}\right)\right)-\boldsymbol{\Sigma}_{v}^{-1 / 2} \psi\left(\boldsymbol{\Sigma}_{v}^{-1 / 2} \boldsymbol{v}\right)=0 .
$$

The non-robust estimates $\hat{\boldsymbol{\beta}}_{\left(u_{i j}\right)}^{g w}$ and $\hat{\boldsymbol{v}}^{g w}$ in the GWEBLUP (4) will be substituted by their robust versions $\hat{\boldsymbol{\beta}}_{\left(u_{i j}\right)}^{\psi, g w}$ and $\hat{\boldsymbol{v}}^{\psi, g w}$ defined as the solutions of the estimating equations (6) and (9). The RGWEBLUP for 
the mean $\bar{y}_{i}$ in area $i$ is then

$$
\hat{\bar{y}}_{i}^{R G W E B L U P}=N_{i}^{-1}\left\{\sum_{j \in s_{i}} y_{i j}+\sum_{j \in r_{i}} \hat{y}_{i j}^{\psi, g w}\right\}=N_{i}^{-1}\left\{\sum_{j \in s_{i}} y_{i j}+\sum_{j \in r_{i}}\left(\boldsymbol{x}_{i j}^{T} \hat{\boldsymbol{\beta}}_{\left(u_{i j}\right)}^{\psi, g w}+\hat{v}_{i}^{\psi, g w}\right)\right\} .
$$

However, the robust estimates $\hat{\boldsymbol{\beta}}_{\left(u_{i j}\right)}^{\psi, g w}$ and $\hat{\boldsymbol{v}}^{\psi, g w}$ cannot be represented in a closed form. We propose an iterative algorithm to estimate $\boldsymbol{\beta}_{\left(u_{i j}\right)}$ and $\boldsymbol{\theta}$ jointly from the equations (6) and (8). For the estimation of $\boldsymbol{\beta}_{\left(u_{i j}\right)}$, we first used a Newton-Raphson algorithm similar to Sinha and Rao (2009). We experienced that the Newton-Raphson algorithm performed unstable in some situations due to the geographical weights. Therefore, we applied a Fix-Point algorithm for the estimation of the $\boldsymbol{\beta}_{\left(u_{i j}\right)}$ in equation (6) which is shortly outlined in Appendix. The equation (8) for the variance components $\boldsymbol{\theta}$ is solved following the ideas of Chatrchi (2012). Afterwards, the robust random effects $\hat{\boldsymbol{v}}^{\psi, g w}$ are obtained from equation (9) by a Fisher-Scoring algorithm. The steps of the algorithm for the estimation of $\boldsymbol{\beta}_{\left(u_{i j}\right)}, \boldsymbol{\theta}$ and $\boldsymbol{v}$ are outlined in the Appendix.

\subsection{Bias correction for the robust GWEBLUP}

The presented estimators in Subsection 2.2 assume that the non-sampled individuals in the population follow exactly the model (3). This assumption implies that departures from the model caused by outlier contamination affects exclusively the sample and not the non-sampled units of the population. Chambers (1986) called this type of contamination as non-representative outliers. In the case of non-representative outlier contamination the estimators introduced in Subsection 2.2 produce unbiased estimates of the small area means (Sinha and Rao, 2009). Chambers et al. (2014) refers to these estimators, such as the REBLUP (5) and the RGWEBLUP (10), as robust projective. However, the concept of non-representative outliers may be violated in real situations and the robust projective estimators can be severely biased. Outliers which affect the sampled and non-sampled part of the population are referred to representative outliers (Chambers, 1986). To account for the presence of representative outliers in the population Chambers et al. (2014) suggested to add a bias correction term to the robust projective estimators. Such bias-corrected estimators are denoted as robust predictive because they try to predict the contribution of the outliers in the population to the small area means.

Chambers et al. (2014) introduced a robust predictive extension of the REBLUP (5) for the mean $\bar{y}_{i}$ in area $i$ by

$$
\hat{\bar{y}}_{i}^{R E B L U P-b c}=\hat{\bar{y}}_{i}^{R E B L U P}+b c_{i}
$$

with

$$
b c_{i}=\left(\frac{N_{i}-n_{i}}{N_{i}}\right) n_{i}^{-1} \sum_{j \in s_{i}} \omega_{i}^{\psi} \phi\left\{\left(y_{i j}-\mathbf{x}_{i j} \hat{\boldsymbol{\beta}}^{\psi}-\hat{v}_{i}^{\psi}\right) / \omega_{i}^{\psi}\right\}
$$

where $\omega_{i}^{\psi}$ is a robust estimator of the scale of the residuals in area $i$. The function $\phi$ is defined by Huber's influence function with a tuning constant $h$. For details we refer to Chambers et al. (2014).

In order to develop a bias correction for GWEBLUP (10) we followed the ideas of Chambers et al. (2014) and additionally accounted for the geographical weighting leading to robust-predictive RGWE- 
BLUP for the mean $\bar{y}_{i}$ in area $i$ by

$$
\hat{\bar{y}}_{i}^{R G W E B L U P-b c}=\hat{\bar{y}}_{i}^{R G W E B L U P}+b c_{i}^{g w}
$$

with

$$
b c_{i}^{g w}=\left(\frac{N_{i}-n_{i}}{N_{i}}\right) \frac{1}{\sum_{j \in s_{i}} \bar{w}_{i j}} \sum_{j \in s_{i}} \bar{w}_{i j} \omega_{i}^{\psi, g w} \phi\left\{\left(y_{i j}-\mathbf{x}_{i j} \hat{\boldsymbol{\beta}}_{\left(u_{i j}\right)}^{\psi, g w}-\hat{v}_{i}^{\psi, g w}\right) / \omega_{i}^{\psi, g w}\right\}
$$

where $\bar{w}_{i j}=n_{i}^{-1} \sum_{k \in s_{i}} w\left(u_{i j}, u_{k}\right)$ is the average geographical weight of the sampled observation $j$ in area $i$. If only the coordinates of centroid for area $i$ are available the geographically weighted biascorrection (14) reduces to (12) because $\bar{w}_{i j}=1$ for all sampled observations in area $i$.

Note that we also explored other possibilities to incorporate the geographical weights into the bias correction by (i) using the median instead if the mean geographical weight in equation (14), (ii) using the mean/median of the out of sample geographical weights from step 10 in the algorithm (Appendix B) and compute a weighted sum over all individual bias corrections from the out of sample predictions, (iii) ignoring the geographical weights and directly use the bias correction from equation (12). However, an empirical study showed that the proposed bias correction from equation (14) appeared to have the best properties in terms of bias and efficiency. The results from the empirical study are available from the authors upon request.

We employ in this paper only one possible way of correcting the bias of robust projective estimators. We are aware of alternative ways proposed by Dongmo-Jiongo et al. (2013). They mentioned that the bias-corrections (11) and (14) may suffer from high variability because the bias-correction depends only on local information in area $i$ which is usually small. Dongmo-Jiongo et al. (2013) introduced an alternative robust predictive estimator that use a global bias-correction which is based on information from other areas. Schmid et al. (2016) compared the robust projective estimators proposed by DongmoJiongo et al. (2013) and Chambers et al. (2014) under spatially correlated random effects. However, the extension of the global bias-corrections proposed by Dongmo-Jiongo et al. (2013) to the case of spatial non-stationarity is currently an open research problem.

\section{Mean squared error estimation}

In this section we introduce two analytic approaches of MSE estimation for the proposed robust estimators. In particular, we propose in Section 3.1 an MSE estimator for the robust projective RGWEBLUP (10) based on a pseudo-linearization approach introduced by Chambers et al. (2011). In Section 3.2 we use first-order approximations for the variance components and propose an MSE estimator for the RGWEBLUP (10) based on a full-linearization suggested by Chambers et al. (2014). These analytic MSE estimators account for the spatial structure in the data. Afterwards we extend both MSE estimators to the robust predictive estimator RGWEBLUP-bc (13). 


\subsection{MSE estimation based on pseudo-linearization}

The MSE estimation suggested by Chambers et al. (2011) is based on a pseudo-linearization approach. In particular, the small area estimator of interest can be written as a weighted sum of the sample values of the target variable. We apply the pseudo-linearization approach to develop a conditional MSE estimation for the RGWEBLUP (10). Conditional in that sense that we treat the random effects to be fixed, but unknown. We show in the Appendix that the RGWEBLUP of $\bar{y}_{i}$ can be expressed as

$$
\hat{\bar{y}}_{i}^{R G W E B L U P}=\sum_{j \in s} d_{i j}^{R G W E B L U P} y_{j}=\left(\boldsymbol{d}_{i}^{R G W E B L U P}\right)^{T} \boldsymbol{y}
$$

where

$$
\left(\boldsymbol{d}_{i}^{R G W E B L U P}\right)^{T}=N_{i}^{-1}\left[\boldsymbol{\delta}_{i}^{T}+\left(N_{i}-n_{i}\right)\left(\overline{\boldsymbol{\nu}}_{i}+\overline{\boldsymbol{\xi}}_{i}\right)\right]
$$

$\boldsymbol{\delta}_{i}$ is an indicator vector of size $n$ which is 1 for the sampled units in area $i$ and is 0 otherwise. $\overline{\boldsymbol{\nu}}_{i}$ and $\overline{\boldsymbol{\xi}}_{i}$ are $1 \times n$ vectors defined by

1. $\overline{\boldsymbol{\nu}}_{i}=\left(N_{i}-n_{i}\right)^{-1} \sum_{j \in r_{i}} \boldsymbol{\nu}_{i j}=\left(N_{i}-n_{i}\right)^{-1} \sum_{j \in r_{i}} \boldsymbol{x}_{i j}^{T}\left(\boldsymbol{X}^{T} \hat{\boldsymbol{V}}_{i j}^{-1} \boldsymbol{X}\right)^{-1} \boldsymbol{X}^{T} \hat{\boldsymbol{V}}_{i j}^{-1} \boldsymbol{D}_{1}$ with $\boldsymbol{D}_{1}$ being a $n \times n$ diagonal matrix of weights $d_{1, i j}=y_{i j}^{*} / y_{i j}$ where the values $y_{i j}^{*}$ are truncated sampled observations of $y_{i j}$ with

$$
y_{i j}^{*}=y_{i j} \min \left(1, \frac{b \sqrt{\hat{\sigma}_{v}^{2}+\hat{\sigma}_{e}^{2}}}{\left|y_{i j}\right|}+\boldsymbol{x}_{i j}^{T} \hat{\boldsymbol{\beta}}_{\left(u_{i j}\right)}^{\psi, g w}\right) .
$$

2. $\overline{\boldsymbol{\xi}}_{i}=\left(N_{i}-n_{i}\right)^{-1} \sum_{j \in r_{i}} \boldsymbol{\xi}_{i j}=\left(N_{i}-n_{i}\right)^{-1} \sum_{j \in r_{i}} \boldsymbol{z}_{i j}^{T} \boldsymbol{Q}\left(\boldsymbol{I}_{n}-\boldsymbol{H}\right)$ where

$$
\boldsymbol{Q}=\hat{\boldsymbol{\Sigma}}_{v}^{1 / 2} \boldsymbol{D}_{3} \hat{\boldsymbol{\Sigma}}_{v}^{1 / 2} \boldsymbol{Z}^{T}\left(\boldsymbol{Z} \hat{\boldsymbol{\Sigma}}_{v}^{1 / 2} \boldsymbol{D}_{3} \hat{\boldsymbol{\Sigma}}_{v}^{1 / 2} \boldsymbol{Z}^{T}+\hat{\boldsymbol{\Sigma}}_{e}^{1 / 2} \boldsymbol{D}_{2} \hat{\boldsymbol{\Sigma}}_{e}^{1 / 2}\right)^{-1}
$$

$\boldsymbol{D}_{2}$ and $\boldsymbol{D}_{3}$ are $n \times n$ and $m \times m$ diagonal matrices, respectively, with elements

$$
\begin{aligned}
d_{2, i j} & =\psi\left\{\left(\hat{\sigma}_{e}^{\psi, g w}\right)^{-1}\left(y_{i j}-\mathbf{x}_{i j} \hat{\boldsymbol{\beta}}_{\left(u_{i j}\right)}^{\psi, g w}-\hat{v}_{i}^{\psi, g w}\right)\right\} /\left\{\left(\hat{\sigma}_{e}^{\psi, g w}\right)^{-1}\left(y_{i j}-\mathbf{x}_{i j} \hat{\boldsymbol{\beta}}_{\left(u_{i j}\right)}^{\psi}-\hat{v}_{i}^{\psi, g w}\right)\right\} \\
d_{3, i} & =\psi\left\{\left(\hat{\sigma}_{u}^{\psi, g w}\right)^{-1} \hat{v}_{i}^{\psi, g w}\right\} /\left\{\left(\hat{\sigma}_{u}^{\psi, g w}\right)^{-1} \hat{v}_{i}^{\psi, g w}\right\}
\end{aligned}
$$

and $\boldsymbol{H}$ is an $n \times n$ projection matrix with row vectors $\boldsymbol{h}_{i j}$ given by

$$
\boldsymbol{h}_{i j}=\boldsymbol{x}_{i j}^{T}\left(\boldsymbol{X}^{T} \hat{\boldsymbol{V}}_{i j}^{-1} \hat{\boldsymbol{U}}_{i j}^{1 / 2} \boldsymbol{D}_{4(i j)} \hat{\boldsymbol{U}}_{i j}^{-1 / 2} \boldsymbol{X}\right)^{-1} \boldsymbol{X}^{T} \hat{\boldsymbol{V}}_{i j}^{-1} \hat{\boldsymbol{U}}_{i j}^{1 / 2} \boldsymbol{D}_{4(i j)} \hat{\boldsymbol{U}}_{i j}^{-1 / 2}
$$

where $\boldsymbol{D}_{4(i j)}$ is an $n \times n$ diagonal matrix with

$$
\operatorname{diag}\left(\boldsymbol{D}_{4(i j)}\right)=\psi\left\{\hat{\boldsymbol{U}}_{i j}^{-1 / 2}\left(\boldsymbol{y}-\boldsymbol{X} \hat{\boldsymbol{\beta}}_{\left(u_{i j}\right)}^{\psi, g w}\right)\right\} / \hat{\boldsymbol{U}}_{i j}^{-1 / 2}\left(\boldsymbol{y}-\boldsymbol{X} \hat{\boldsymbol{\beta}}_{\left(u_{i j}\right)}^{\psi, g w}\right)
$$

We use expression (15) to develop an approximation for the conditional MSE for the RGWEBLUP (10). According to the surnames of the authors who contributed to Chambers et al. (2011) we will refer to this 
method by adding the subscript CCT. The estimator of the conditional MSE for the RGWEBLUP of $\bar{y}_{i}$ is then

$$
\widehat{M S E}_{C C T}\left(\hat{\bar{y}}_{i}^{R G W E B L U P}\right)=\widehat{\operatorname{Var}}_{C C T}\left(\hat{\bar{y}}_{i}^{R G W E B L U P}\right)+\widehat{\operatorname{Bias}}\left(\hat{\bar{y}}_{i}^{R G W E B L U P}\right)^{2}
$$

where

$$
\widehat{\operatorname{Var}}_{C C T}\left(\hat{\bar{y}}_{i}^{R G W E B L U P}\right)=N_{i}^{-2} \sum_{j \in s}\left\{a_{i j}^{2}+\left(N_{i}-n_{i}\right) n^{-1}\right\} \hat{\lambda}_{j}^{-1}\left(y_{j}-\hat{\mu}_{j}\right)^{2}
$$

is an estimate of the conditional prediction variance of (10), with $a_{i j}=N_{i} d_{i j}^{R G W E B L U P}-I(j \in i)$. $I(j \in i)$ is an indicator function which is equal to 1 whenever unit $j$ is in area $i$. The second term on the right hand side of (18)

$$
\widehat{\operatorname{Bias}}\left(\hat{\bar{y}}_{i}^{R G W E B L U P}\right)=\sum_{j \in s} d_{i j}^{R G W E B L U P} \hat{\mu}_{j}-N_{i}^{-1} \sum_{j \in N_{i}} \hat{\mu}_{j}
$$

denotes an estimate of the conditional prediction bias of (10). Here $\hat{\mu}_{j}=\sum_{k \in s} \gamma_{k j} y_{k}=\gamma_{j}^{T} \boldsymbol{y}$ is an unbiased linear estimator of the conditional expected value $\mu_{j}=E\left(y_{j} \mid \boldsymbol{x}_{j}, \boldsymbol{v}^{\psi}\right)$ where under model (3) $\gamma_{j}^{T}=\left(\boldsymbol{\nu}_{i j}+\boldsymbol{\xi}_{i j}\right)$ for $j \in r_{i}$ and $\gamma_{j}^{T}=\left(\boldsymbol{h}_{i j}+\boldsymbol{\xi}_{i j}\right)$ for $j \in s_{i}$. For additional details we refer to Chambers et al. (2011). Further, $\hat{\lambda}_{j}=1-2 \gamma_{j j}+\sum_{k \in s} \gamma_{k j}^{2}$ is defined as a scaling constant. However, due to the shrinkage effect which can lead to biased EBLUP estimates, Chambers et al. (2011) recommended to compute $\hat{\mu}_{j}$ as the 'unshrunken' version. Following that suggestion $\boldsymbol{\xi}_{i j}$ reduces to $\boldsymbol{\xi}_{i j}^{u}=\boldsymbol{z}_{i j}^{T}\left(\boldsymbol{Z} \boldsymbol{Z}^{T}\right)^{-1} \boldsymbol{Z}^{T}\left(\boldsymbol{I}_{n}-\boldsymbol{H}\right)$ for the estimation of $\hat{\mu}_{j}$ and, furthermore, $\hat{\lambda}_{j}$ would be of order $1+O\left(n^{-1}\right)$. Thus, we set $\hat{\lambda}_{j} \equiv 1$ in equation (19).

The MSE estimator for the robust predictive RGWEBLUP-bc (13) can be developed using the pseudo-linearization approach introduced above. The weights $d_{i j}^{R G W E B L U P}$ from (15) need to be adjusted for the bias correction. Since RGWEBLUP-bc (13) is approximately unbiased the MSE estimator reduces to

$$
\widehat{M S E}_{C C T}\left(\hat{\bar{y}}_{i}^{R G W E B L U P-b c}\right)=\widehat{\operatorname{Var}}_{C C T}\left(\hat{\bar{y}}_{i}^{R G W E B L U P-b c}\right),
$$

with

$$
\hat{\bar{y}}_{i}^{R G W E B L U P-b c}=\sum_{j \in s} d_{i j}^{R G W E B L U P-b c} y_{j}=\left(\boldsymbol{d}_{i}^{R G W E B L U P-b c}\right)^{T} \boldsymbol{y}
$$

Let $\boldsymbol{q}$ be an $n \times 1$ vector with components

$$
q_{i j}=\frac{\bar{w}_{i j} \omega_{i}^{\psi, g w} \phi\left\{\left(y_{i j}-\mathbf{x}_{i j} \hat{\boldsymbol{\beta}}_{\left(u_{i j}\right)}^{\psi, g w}-\hat{v}_{i}^{\psi, g w}\right) / \omega_{i}^{\psi, g w}\right\}}{\left(y_{i j}-\mathbf{x}_{i j} \hat{\boldsymbol{\beta}}_{\left(u_{i j}\right)}^{\psi, g w}-\hat{v}_{i}^{\psi, g w}\right)}
$$

and $\mathbf{1}_{n}$ be a $n \times 1$ vector of ones. Then expression (14) can be rewritten as

$$
b c_{i}^{g w}=\left(\frac{N_{i}-n_{i}}{N_{i}}\right) \frac{1}{\sum_{j \in s_{i}} \bar{w}_{i j}} \sum_{j \in s_{i}} q_{i j}\left(y_{i j}-\mathbf{x}_{i j} \hat{\boldsymbol{\beta}}_{\left(u_{i j}\right)}^{\psi, g w}-\hat{v}_{i}^{\psi, g w}\right),
$$


leading after some rearrangements to (additional details are provided in the Appendix)

$$
\begin{aligned}
\left(\boldsymbol{d}_{i}^{R G W E B L U P-b c}\right)^{T}= & N_{1}^{-1}\left\{\left(\mathbf{1}_{n}+\frac{N_{i}-n_{i}}{\sum_{j \in s_{i}} \bar{w}_{i j}} \boldsymbol{q}\right)^{T} \circ \boldsymbol{\delta}_{i}^{T}\right. \\
& +\left(N_{i}-n_{i}\right)\left(\overline{\boldsymbol{\nu}}_{i}-\frac{1}{\sum_{j \in s_{i}} \bar{w}_{i j}} \sum_{j \in s_{i}} q_{i j} \boldsymbol{h}_{i j}\right) \\
& \left.+\left(N_{i}-n_{i}\right)\left(\overline{\boldsymbol{\xi}}_{i}-\frac{1}{\sum_{j \in s_{i}} \bar{w}_{i j}} \sum_{j \in s_{i}} q_{i j} \boldsymbol{\xi}_{i j}\right)\right\} .
\end{aligned}
$$

The MSE estimator (21) for the RGWEBLUP-bc (13) can be calculated by plugging the weights (23) into the estimator (19) for the variance component. Note that the MSE estimators for the RGWEBLUP (18) and for the RGWEBLUP-bc (21) ignore the additional uncertainty introduced by the estimation of the variance components and therefore may be seen as a first-order approximation of the MSE.

\subsection{MSE estimation based on linearization}

Following Chambers et al. (2014) we propose a linearization based approach for the MSE estimation of the RGWEBLUP (10) and the RGWEBLUP-bc (13). The basic idea is to decompose the MSE into a squared bias term, the prediction variance and an additional term that accounts for the variability caused by the estimation of the variance components. Like in Subsection 3.1, we develop the MSE estimation conditional on the realization of the random effects. We assume the same regularity conditions as Chambers et al. (2014). Note that the derivations for the MSE estimation in this subsection extend the work of Chambers et al. (2014) and account for the additional spatial structure in the data. To start with we assume that the variance parameters from model (3) are known. The variance of the prediction error of the RGWBLUP $\hat{\bar{y}}_{i}^{R G W B L U P}$ can be expressed as

$$
\begin{aligned}
\operatorname{Var}\left(\hat{\bar{y}}_{i}^{R G W B L U P}-\bar{y}\right)= & \operatorname{Var}\left(N_{i}^{-1} \sum_{j \in r_{i}}\left(\boldsymbol{x}_{i j}^{T} \tilde{\boldsymbol{\beta}}_{\left(u_{i j}\right)}^{\psi, g w}+\tilde{v}_{i}^{\psi, g w}\right)-N_{i}^{-1} \sum_{j \in r_{i}} y_{i j}\right) \\
= & \frac{1}{N_{i}^{2}} \operatorname{Var}\left(\sum_{j \in r_{i}} \boldsymbol{x}_{i j}^{T} \tilde{\boldsymbol{\beta}}_{\left(u_{i j}\right)}^{\psi, g w}\right)+\left(\frac{N_{i}-n_{i}}{N_{i}}\right)^{2} \operatorname{Var}\left(\tilde{v}_{i}^{\psi, g w}\right) \\
& +\left(\frac{N_{i}-n_{i}}{N_{i}}\right)^{2} \operatorname{Var}\left(\bar{e}_{r i}\right)
\end{aligned}
$$

where $\tilde{\boldsymbol{\beta}}_{\left(u_{i j}\right)}^{\psi, g w}$ and $\tilde{v}_{i}^{\psi, g w}$ are assumed to be independent and $\bar{e}_{r i}=\left(N_{i}-n_{i}\right)^{-1} \sum_{j \in r_{i}}\left(y_{i j}-\boldsymbol{x}_{i j}^{T} \tilde{\boldsymbol{\beta}}_{\left(u_{i j}\right)}^{\psi, g w}-\right.$ $\left.\tilde{v}_{i}^{\psi, g w}\right)$. Note that $\tilde{\boldsymbol{\beta}}_{\left(u_{i j}\right)}^{\psi, g w}$ and $\tilde{v}_{i}^{\psi, g w}$ are solutions to the equations (6) and (9) when the variance compo- 
nents are known. The first variance component in (24) can be rewritten as

$$
\begin{aligned}
\frac{1}{N_{i}^{2}} \operatorname{Var}\left(\sum_{j \in r_{i}} \boldsymbol{x}_{i j}^{T} \tilde{\boldsymbol{\beta}}_{\left(u_{i j}\right)}^{\psi, g w}\right) & =\frac{1}{N_{i}^{2}} \sum_{j \in r_{i}} \sum_{k \in r_{i}} \boldsymbol{x}_{i k}^{T} \operatorname{Cov}\left(\tilde{\boldsymbol{\beta}}_{\left(u_{i k}\right)}^{\psi, g w}, \tilde{\boldsymbol{\beta}}_{\left(u_{i j}\right)}^{\psi, g w}\right) \boldsymbol{x}_{i j} \\
& =\frac{1}{N_{i}^{2}} \sum_{j \in r_{i}} \sum_{k \in r_{i}} \boldsymbol{x}_{i k}^{T} \operatorname{Var}\left(\tilde{\boldsymbol{\beta}}_{\left(u_{i j}\right)}^{\psi, g w}\right) \boldsymbol{x}_{i j} \\
& =\left(\frac{N_{i}-n_{i}}{N_{i}^{2}}\right) \overline{\boldsymbol{x}}_{r i}^{T} \sum_{j \in r_{i}} \operatorname{Var}\left(\tilde{\boldsymbol{\beta}}_{\left(u_{i j}\right)}^{\psi, g w}\right) \boldsymbol{x}_{i j}
\end{aligned}
$$

under the condition that $\operatorname{Cov}\left(\tilde{\boldsymbol{\beta}}_{\left(u_{i j}\right)}^{\psi, g w}, \tilde{\boldsymbol{\beta}}_{\left(u_{i k}\right)}^{\psi, g w}\right)=0$ for $j \neq k . \overline{\boldsymbol{x}}_{r i}$ is the p-vector of area specific means from the non-sampled observations. It follows that the MSE estimation of the RWGBLUP depends on $\operatorname{Var}\left(\tilde{\boldsymbol{\beta}}_{\left(u_{i j}\right)}^{\psi, g w}\right), \operatorname{Var}\left(\tilde{v}_{i}^{\psi, g w}\right)$ and $\operatorname{Var}\left(\bar{e}_{r i}\right)$.

Following the estimation algorithm outlined in the Appendix the elements of coefficient matrix $\boldsymbol{B}_{r}$ have the explicit form $\tilde{\boldsymbol{\beta}}_{\left(u_{i j}\right)}^{\psi, g w}=\left(\boldsymbol{X}^{T} \hat{\boldsymbol{V}}_{i j}^{-1} \boldsymbol{X}\right)^{-1} \boldsymbol{X}^{T} \hat{\boldsymbol{V}}_{i j}^{-1} \boldsymbol{y}^{*}$ and the variance directly follows as

$$
\widehat{\operatorname{Var}}_{r}\left(\tilde{\boldsymbol{\beta}}_{\left(u_{i j}\right)}^{\psi, g w}\right)=\left(\boldsymbol{X}^{T} \boldsymbol{V}_{i j}^{-1} \boldsymbol{X}\right)^{-1} \boldsymbol{X}^{T} \boldsymbol{V}_{i j}^{-1} \operatorname{Var}\left(\boldsymbol{y}^{*}\right) \boldsymbol{V}_{i j}^{-1} \boldsymbol{X}\left(\boldsymbol{X}^{T} \boldsymbol{V}_{i j}^{-1} \boldsymbol{X}\right)^{-1}
$$

where $\operatorname{Var}\left(\boldsymbol{y}^{*}\right)=\boldsymbol{V}$. To find asymptotic solutions for $\operatorname{Var}\left(\tilde{v}_{i}^{\psi, g w}\right)$ and the variance of the in-sample coefficients $\boldsymbol{B}_{s}$ we use of the estimating equation (6) and (9) which lead to sandwich-type estimators

$$
\begin{aligned}
\widehat{\operatorname{Var}}\left(\tilde{v}_{i}^{\psi, g w}\right) & =\boldsymbol{\Xi}^{-1}\left(\nu(\boldsymbol{t}) \boldsymbol{Z}^{T} \hat{\boldsymbol{\Sigma}}_{e}^{-1} \boldsymbol{Z}\right)\left[\boldsymbol{\Xi}^{-1}\right]^{T} \\
\widehat{\operatorname{Var}}_{s}\left(\tilde{\boldsymbol{\beta}}_{\left(u_{i j}\right)}^{\psi, g w}\right) & =\boldsymbol{\Pi}^{-1}\left(\nu\left(\boldsymbol{r}_{i j}\right) \boldsymbol{X} \hat{\boldsymbol{V}}_{i j}^{-1} \hat{\boldsymbol{U}}_{i j} \hat{\boldsymbol{V}}_{i j}^{-1} \boldsymbol{X}\right)\left[\boldsymbol{\Pi}^{-1}\right]^{T},
\end{aligned}
$$

where

$$
\begin{aligned}
\boldsymbol{\Xi} & =-\boldsymbol{Z}^{T} \hat{\boldsymbol{\Sigma}}_{e}^{-1 / 2} \boldsymbol{T} \hat{\boldsymbol{\Sigma}}_{e}^{-1 / 2} \boldsymbol{Z}-\hat{\boldsymbol{\Sigma}}_{v}^{-1 / 2} \boldsymbol{L} \hat{\boldsymbol{\Sigma}}_{v}^{-1 / 2} \\
\boldsymbol{\Pi} & =-\boldsymbol{X} \hat{\boldsymbol{V}}_{i j}^{-1} \hat{\boldsymbol{U}}_{i j}^{1 / 2} \boldsymbol{R}_{i j} \hat{\boldsymbol{U}}_{i j}^{1 / 2} \boldsymbol{X} \\
\boldsymbol{t} & =\hat{\boldsymbol{\Sigma}}_{e}^{-1 / 2}\left(\boldsymbol{y}-\boldsymbol{\rho}_{s}-\boldsymbol{Z} \boldsymbol{v}\right)
\end{aligned}
$$

$\boldsymbol{r}_{i j}$ and $\boldsymbol{\rho}_{s}$ are defined as in equations (6) and (8) respectively. In addition, $\boldsymbol{T}=\operatorname{diag}_{n}[I(-c<\boldsymbol{t}<c)]$, $\boldsymbol{L}=\operatorname{diag}_{m}\left[I\left(-c<\hat{\boldsymbol{\Sigma}}_{v}^{-1 / 2} \tilde{\boldsymbol{v}}^{\psi, g w}<c\right)\right]$ and $\boldsymbol{R}_{i j}=\operatorname{diag}_{n}\left[I\left(-c<\boldsymbol{r}_{i j}<c\right)\right] . c$ denotes the tuning constant in Huber's influence function. The function $\nu(\boldsymbol{a})=\left(\eta \sum_{j=1}^{n} \psi^{2}\left(a_{i}\right)\right) /(n-p)$, is an estimator for the variance $\operatorname{Var}(\psi(\boldsymbol{a}))$ with $\eta=\left(1+p \operatorname{Var}\left(\psi^{\prime}(\boldsymbol{a})\right) / n E\left[\psi^{\prime}(\boldsymbol{a})\right]^{2}\right)$ as a bias correction term which was suggested by Huber (1964). Note that the variance of the in-sample coefficients in expression (27) is needed later when we account for the bias correction.

Based on the derivations in the equations (25) and (26), we define the following estimator for the variance of the prediction error (24)

$$
\hat{V}\left(\hat{\bar{y}}_{i}^{R G W B L U P}-\bar{y}_{i}\right)=h_{1 i}\left(\tilde{B}_{r_{i}}^{\psi, g w}, \tilde{v}_{i}^{\psi, g w}\right)+h_{2 i}\left(\tilde{B}_{s}^{\psi, g w}, \tilde{\boldsymbol{v}}^{\psi, g w}\right),
$$


where

1. The first component $h_{1 i}$ is due to the estimation of the regression coefficients and the area specific random effects and is defined by:

$$
h_{1 i}\left(\tilde{B}_{r_{i}}^{\psi, g w}, \tilde{v}_{i}^{\psi, g w}\right)=\left(\frac{N_{i}-n_{i}}{N_{i}^{2}}\right) \overline{\boldsymbol{x}}_{r i}^{T} \sum_{j \in r_{i}} \widehat{\operatorname{Var}}\left(\tilde{\boldsymbol{\beta}}_{\left(u_{i j}\right)}^{\psi, g w}\right) \boldsymbol{x}_{i j}+\left(\frac{N_{i}-n_{i}}{N_{i}}\right)^{2} \widehat{\operatorname{Var}}\left(\tilde{v}_{i}^{\psi, g w}\right) .
$$

2. The second component $h_{2 i}$ can be either estimated using only the area specific sample residuals or by using the residuals from the entire sample. Chambers et al. (2014) recommend to use the latter estimator since it can yield MSE estimates which are more stable when the area specific sample sizes are very small. We will follow that suggestion and define

$$
h_{2 i}\left(\tilde{B}_{s}^{\psi, g w}, \tilde{\boldsymbol{v}}^{\psi, g w}\right)=\left(\frac{N_{i}-n_{i}}{N_{i}}\right)^{2} \widehat{\operatorname{Var}}\left(\hat{\bar{e}}_{r i}\right)=\left(\frac{N_{i}-n_{i}}{(n-1) \cdot N_{i}^{2}}\right) \sum_{i=1}^{m} \sum_{j \in s_{i}}\left(y_{i j}-\boldsymbol{x}_{i j}^{T} \tilde{\boldsymbol{\beta}}_{\left(u_{i j}\right)}^{\psi, g w}-\boldsymbol{z}_{i}^{T} \tilde{\boldsymbol{v}}^{\psi, g w}\right)^{2} .
$$

Using equation (28) the conditional MSE of the RGWBLUP can be estimated by

$$
\widehat{M S E}_{C C S T}\left(\hat{\bar{y}}_{i}^{R G W B L U P}\right)=h_{1 i}\left(\tilde{B}_{r_{i}}^{\psi, g w}, \tilde{v}_{i}^{\psi, g w}\right)+h_{2 i}\left(\tilde{B}_{s}^{\psi, g w}, \tilde{\boldsymbol{v}}^{\psi, g w}\right)+\widehat{\operatorname{Bias}}\left(\hat{\bar{y}}_{i}^{R G W B L U P}\right)^{2} .
$$

Note that according to the surnames of the authors who contributed to Chambers et al. (2014) we refer to this MSE estimator by adding the subscript CCST. Following the ideas of Chambers et al. (2014) we add an extra term to equation (29) in order to account for the extra variability caused by the estimation of the variance components $\boldsymbol{\theta}$. The proposed estimator of the conditional MSE for the RGWEBLUP of $\bar{y}_{i}$ has the form

$$
\begin{aligned}
\widehat{M S E}_{C C S T}\left(\hat{\bar{y}}_{i}^{R G W E B L U P}\right)= & h_{1 i}\left(\hat{B}_{r_{i}}^{\psi, g w}, \hat{v}_{i}^{\psi, g w}\right)+h_{2 i}\left(\hat{B}_{s}^{\psi, g w}, \hat{\boldsymbol{v}}^{\psi, g w}\right) \\
& +h_{3 i}\left(\hat{B}_{s}^{\psi, g w}, \hat{\boldsymbol{v}}^{\psi, g w}\right)+\widehat{\operatorname{Bias}}\left(\hat{\bar{y}}_{i}^{R G W E B L U P}\right)^{2}
\end{aligned}
$$

where $\widehat{\operatorname{Bias}}\left(\hat{\bar{y}}_{i}^{R G W E B L U P}\right)^{2}$ is given by equation (20). The component $h_{3 i}$ is the variability added due to the estimation of the variance components $\boldsymbol{\theta}$ and is defined by

$$
h_{3 i}\left(\hat{B}_{s}^{\psi, g w}, \hat{\boldsymbol{v}}^{\psi, g w}\right)=\left(N_{i}^{-1} \sum_{j \in r_{i}} \boldsymbol{z}_{i j}^{T}\right) \Upsilon \operatorname{Var}(\hat{\boldsymbol{\theta}})\left(N_{i}^{-1} \sum_{j \in r_{i}} \boldsymbol{z}_{i j}^{T}\right)^{T}
$$

with

$$
\Upsilon=\sum_{k=1}^{2} \sum_{g=1}^{2}\left\{\left(\left.\partial \boldsymbol{Q}\right|_{\theta_{k}}\right)\left[\sum_{j=1}^{m} \sum_{l=1}^{m}\left(\boldsymbol{z}_{i j}^{T} \hat{\boldsymbol{v}}^{\psi, g w}\right)\left(\boldsymbol{z}_{i l}^{T} \hat{\boldsymbol{v}}^{\psi, g w}\right)+\sigma_{e}^{2} I(j=l)\right]\left(\left.\partial \boldsymbol{Q}\right|_{\theta_{g}}\right)^{T}\right\}
$$

where $\boldsymbol{Q}$ is defined as in equation (17). For an estimate of the variance-covariance matrix $\operatorname{Var}(\hat{\boldsymbol{\theta}})$ we apply the delta method to the robust estimation equations proposed by Sinha and Rao (2009). 
The linearization approach can also be applied to develop an MSE estimate for the robust predictive extension RGWEBLUP-bc. Again, we will start the derivation by assuming that the variance parameter $\boldsymbol{\theta}$ from model (3) is known. The prediction error for RGWBLUP-bc of $\bar{y}_{i}$ can be written as

$$
\hat{\bar{y}}_{i}^{R G W B L U P-b c}-\bar{y}_{i}=N_{i}^{-1} \sum_{j \in r_{i}}\left(\boldsymbol{x}_{i j}^{T} \tilde{\boldsymbol{\beta}}_{\left(u_{i j}\right)}^{\psi, g w}+\tilde{v}_{i}^{\psi, g w}\right)+b c_{i}^{g w}-\bar{y}_{i} .
$$

After applying a first order Taylor series approximation to the latter expression an estimator of the conditional the MSE for the RGWBLUP-bc can be approximated analogously to equation (29) by

$$
\widehat{M S E}_{C C S T}\left(\hat{\bar{y}}_{i}^{R G W B L U P-b c}\right)=h_{1 i}^{b c}\left(\tilde{B}_{r_{i}}^{\psi, g w}, \tilde{v}_{i}^{\psi, g w}\right)+h_{2 i}\left(\tilde{B}_{s}^{\psi, g w}, \tilde{\boldsymbol{v}}^{\psi, g w}\right)+h_{4 i}^{b c}\left(\tilde{B}_{s_{i}}^{\psi, g w}, \tilde{v}_{i}^{\psi, g w}\right)
$$

where the first term on the right hand side can be expressed as

$$
h_{1 i}^{b c}\left(\tilde{\boldsymbol{\beta}}_{\left(u_{i j}\right)}^{\psi, g w}\right)=\left(1-n_{i} N_{i}^{-1}\right)^{2}\left(\bar{\Lambda}_{r i}-\bar{\Lambda}_{s i}\right)\left(\overline{\boldsymbol{x}}_{r i}-\overline{\boldsymbol{x}}_{s i}^{*}\right)
$$

with $\bar{\Lambda}_{r i}=\left(N_{i}-n_{i}\right)^{-1} \sum_{j \in r_{i}} \boldsymbol{x}_{i j} \widehat{\operatorname{Var}}\left(\tilde{\boldsymbol{\beta}}_{\left(u_{i j}\right)}^{\psi, g w}\right)$ and $\bar{\Lambda}_{s i}=n_{i}^{-1} \sum_{j \in s_{i}} \boldsymbol{x}_{i j}^{*} \widehat{\operatorname{Var}}\left(\tilde{\boldsymbol{\beta}}_{\left(u_{i j}\right)}^{\psi, g w}\right)$. The star in the upper expression refers to the transformation $f(a)=\frac{n \bar{w}_{i j}}{\sum_{j \in s_{i}} \bar{w}_{i j}} a$ and $\widehat{\operatorname{Var}}\left(\tilde{\boldsymbol{\beta}}_{\left(u_{i j}\right)}^{\psi, g w}\right)$ is defined by equation (27). The second term of (31) is equivalent to $h_{2 i}$ defined in equation (29) and the third term is given by

$$
h_{4 i}^{b c}\left(\tilde{B}_{s_{i}}^{\psi, g w}, \tilde{v}_{i}^{\psi, g w}\right)=\frac{\left(1-n_{i} N_{i}^{-1}\right)^{2}}{\left(g_{1 i}\left(g_{1 i}-g_{2 i} / g_{1 i}\right)\right)} \sum_{j \in s_{i}} \bar{w}_{i j}\left(\omega_{i}^{\psi, g w} \phi\left\{\left(y_{i j}-\boldsymbol{x}_{i j}^{T} \tilde{\boldsymbol{\beta}}_{\left(u_{i j}\right)}^{\psi, g w}-\tilde{v}_{i}^{\psi, g w}\right) / \omega_{i}^{\psi, g w}\right\}\right)^{2}
$$

with $g_{1 i}=\sum_{j \in s_{i}} \bar{w}_{i j}$ and $g_{2 i}=\sum_{j \in s_{i}} \bar{w}_{i j}^{2}$. The conditional MSE estimator of the RGWEBLUP-bc which takes into account the extra variability coming from the estimation of the variance parameters can be obtained by adding an additional term $h_{3 i}^{b c}$ to equation (31), leading to

$$
\begin{aligned}
\widehat{M S E}_{C C S T}\left(\hat{\bar{y}}_{i}^{R G W E B L U P-b c}\right)= & h_{1 i}^{b c}\left(\hat{B}_{r_{i}}^{\psi, g w}, \hat{v}_{i}^{\psi, g w}\right)+h_{2 i}\left(\hat{B}_{s}^{\psi, g w}, \hat{\boldsymbol{v}}^{\psi, g w}\right)+h_{3 i}^{b c}\left(\hat{B}_{s}^{\psi, g w}, \hat{\boldsymbol{v}}^{\psi, g w}\right) \\
& +h_{4 i}^{b c}\left(\hat{B}_{s_{i}}^{\psi, g w}, \hat{v}_{i}^{\psi, g w}\right)
\end{aligned}
$$

where $h_{3 i}^{b c} \approx\left(1-n_{i} N_{i}^{-1}\right) \boldsymbol{\rho}_{i}^{T} \Upsilon \operatorname{Var}(\hat{\theta}) \boldsymbol{\rho}_{i}^{T}$ accounts for the variance added due to the estimation of $\boldsymbol{\theta}$ and $\boldsymbol{\rho}_{i}^{T}=\overline{\boldsymbol{z}}_{r i}-\frac{1}{\sum_{j \in s_{i}} \bar{w}_{i j}} \sum_{j \in s_{i}} \bar{w}_{i j} \phi^{\prime}\left\{\left(y_{i j}-\boldsymbol{x}_{i j}^{T} \hat{\boldsymbol{\beta}}_{\left(u_{i j}\right)}^{\psi, g w}-\hat{v}_{i}{ }^{\psi, g w}\right) / \omega_{i}^{\psi, g w}\right\} \boldsymbol{z}_{i j}$. Note that $\boldsymbol{\rho}_{i}=0$ when $\phi$ is the identity function and the underlying model (3) simplifies to the random intercept model. Since the RGWEBLUP-bc is asymptotically unbiased, the bias term is not needed for the MSE estimator. The MSE estimation of the non-robust GWEBLUP estimator of $\bar{y}_{i}$ can be obtained by setting the tuning constant $b$ to a very high value, i.e. $b=100$, for both, the CCT and the CCST approach. The proposed MSE estimators for robust GWEBLUP approaches (RGWEBLUP and RGWEBLUP-bc) are a generalization of Chambers et al. (2014). By setting the spatial weights in model (3) to be constant for all population units we obtain the results of Chambers et al. (2014). 


\section{Model based simulation}

In this section, we provide results from a model-based simulation study assessing the performance of the robust estimators that were described in Section 2. The scenarios in the model-based simulations are a combination of settings under spatial stationarity and non-stationarity with different outlier contamination mechanisms. We further investigate the performance of the proposed MSE estimators introduced in Section 3.

Following Chambers et al. (2014), we generated population data for $m=40$ small areas. The samples were selected from the population by simple random sampling without replacement within each area. The population and sample sizes were held fixed for all areas at $N_{i}=100$ and $n_{i}=5$ respectively. The covariates $x_{i j}$ were drawn from a log-normal distribution with mean 1 and a standard deviation of 0.5 . We generated the dependent variable $y_{i j}$ as follows:

$$
y_{i j}=\beta_{0(i j)}+\beta_{1(i j)} x_{i j}+v_{i}+e_{i j}
$$

with

$$
\begin{aligned}
& \beta_{0(i j)}=100+a_{0}\left(\text { longitude }_{i j}+\text { latitude }_{i j}\right) \\
& \beta_{1(i j)}=5+a_{1}\left(\text { longitude }_{i j}+\text { latitude }_{i j}\right) .
\end{aligned}
$$

Following Chandra et al. (2012), the parameters $a_{0}$ and $a_{1}$ are set to 0 for spatial stationarity scenarios and to $a_{0}=0.1$ and $a_{1}=0.2$ for scenarios under spatial non-stationarity. The coordinates for the population units where generated as a uniform grid with range $(0, \sqrt{N} / 2)$ for the longitude and the latitude. The random effects $v_{i}$ and the individual error $e_{i j}$ were independently generated under different outlier contamination mechanisms:

1. $(0,0)$ - no outliers, $e \sim N(0,6)$ and $v_{i} \sim N(0,3)$;

2. $(v, e)_{s}$ - symmetric outliers in area-level and individual error, $v_{i} \sim N(0,3)$ for the areas 1-36 and $v_{i} \sim N(0,20)$ for the areas 37-40; $e \sim \delta N(0,6)+(1-\delta) N(0,150)$ where $\delta$ is Bernoulli distributed with $P(\delta=1)=0.95$;

3. $(v, e)_{n s}$ - non-symmetric outliers in area-level and individual error, $v_{i} \sim N(0,3)$ for the areas 1-36 and $v_{i} \sim N(9,20)$ for the areas 37-40; $e \sim \delta N(0,6)+(1-\delta) N(20,150)$ where $\delta$ is Bernoulli distributed with $P(\delta=1)=0.95$.

In particular, the scenario $(0,0)$ serves as a reference when the assumptions of the model (1) are valid. Following Sinha and Rao (2009), we concentrate in the second scenario $(v, e)_{s}$ on symmetric outlier contamination in the area and unit level term. We expect that the outlier robust projective estimators are superior under these settings. However, the assumption of perfect symmetric outlier contamination may be violated in many real applications. Thus, following Chambers et al. (2014), we investigate nonsymmetric outlier contamination in the third scenario $(v, e)_{n s}$. We expect that outlier robust projective estimators suffer here from a potential bias. The outlier robust predictive estimators should be able 
to reduce the bias under this scenario. The choice of the simulation setup (number of area, sample and population sizes) and the outlier contamination mechanism is for two reasons: First, we aimed to investigate the proposed methods for small area estimation under extreme situations. Second, we wanted to have comparable results to Sinha and Rao (2009) and Chambers et al. (2014).

We independently repeated each scenario $T=500$ times. For each Monte-Carlo replication we generated the population according to the underlying setting, drew a sample and used the sampled data to calculate estimates for the area means.

We evaluated six estimators of the small area population averages in the simulations. These are the EBLUP (2), the robust projective REBLUP (5) and the robust predictive REBLUP-bc (11). These estimators are suitable under spatial stationarity because they assume a global model (1). In the following we will refer to them as global estimators. Furthermore, we evaluated their extensions to spatial non-stationarity, the GWEBLUP (4), the robust projective RGWEBLUP (10) and the robust predictive RGWEBLUP-bc (13). Following Dongmo-Jiongo et al. (2013) and Chambers et al. (2014), we set the tuning constant for the bias correction to 3 . The estimators are implemented by computationally efficient algorithms using $\mathrm{C}++$ and $\mathrm{R}$. The codes are available from the authors upon request.

The performance of the estimators was evaluated by the median values of the area specific relative bias (RB) and relative root mean squared errors (RRMSE) given by

$$
\begin{aligned}
\operatorname{RB}\left(\hat{m}_{i}\right) & =\frac{1}{T} \sum_{t=1}^{T} \frac{\hat{m}_{t i}-m_{t i}}{m_{t i}} \\
\operatorname{RRMSE}\left(\hat{m}_{i}\right) & =\sqrt{\frac{1}{T} \sum_{t=1}^{T}\left(\frac{\hat{m}_{t i}-m_{t i}}{m_{t i}}\right)^{2}},
\end{aligned}
$$

where $\hat{m}_{i}$ is generic notation to denote a small area estimator of the average in area $i$ and $m_{i}$ denotes the true population mean in area $i$.

Table 1 presents the median values of the RB and the RRMSE for the spatial stationary and nonstationary scenarios. Starting with the (stationary and non-stationary) scenarios without outliers $(0,0)$, we note that all estimators are almost unbiased. Furthermore, the geographically weighted approaches (GWEBLUP, RGWEBLUP and RGWEBLUP-bc) are more efficient compared to the global approaches (EBLUP, REBLUP and REBLUP-bc) under spatial non-stationarity.

The results for the scenarios with symmetric outlier contamination $(v, e)_{s}$ confirm our expectations regarding the behaviour of the robust estimators. First, the robust projective estimators (REBLUP and RGWEBLUP) lead on average to more efficient results compared to the robust predictive estimators (REBLUP-bc and GWEBLUP-bc) and compared to the non-robust estimators (EBLUP and GWEBLUP) in the corresponding stationary and non-stationary scenarios (areas 1-40). Second, the bias is almost negligible under symmetric outlier contamination, especially in the areas 1-36. Third, the geographically weighted methods offer some gains in RRMSE when compared to the global methods in the spatial non-stationarity scenarios.

In the case of asymmetric outlier contamination $(v, e)_{n s}$ the robust projective estimators (REBLUP 
Table 1: Performance of estimated small area means in the model-based simulation

\begin{tabular}{|c|c|c|c|c|c|c|c|}
\hline \multirow[t]{3}{*}{ Predictor } & \multicolumn{7}{|c|}{ Results (\%) for the following scenarios and areas } \\
\hline & $(0,0)$ & $(v, e)_{s}$ & $(v, e)_{s}$ & $(v, e)_{s}$ & $(v, e)_{n s}$ & $(v, e)_{n s}$ & $(v, e)_{n s}$ \\
\hline & $1-40$ & $1-40$ & $1-36$ & $37-40$ & $1-40$ & $1-36$ & $37-40$ \\
\hline & \multicolumn{7}{|c|}{ Spatial non-stationarity } \\
\hline \multicolumn{8}{|c|}{ Median values of $R B$} \\
\hline GWEBLUP & 0.00 & 0.02 & 0.05 & -0.30 & 0.14 & 0.16 & -1.68 \\
\hline RGWEBLUP & -0.00 & 0.02 & 0.04 & -0.27 & -0.41 & -0.38 & -1.66 \\
\hline RGWEBLUP-bc & 0.01 & 0.00 & 0.02 & -0.16 & -0.45 & -0.43 & -1.16 \\
\hline EBLUP & 0.02 & 0.01 & 0.05 & -0.63 & 0.04 & 0.13 & -1.03 \\
\hline REBLUP & 0.02 & -0.01 & 0.04 & -0.90 & -0.40 & -0.35 & -1.23 \\
\hline REBLUP-bc & 0.01 & 0.03 & 0.03 & -0.47 & -0.40 & -0.35 & -0.73 \\
\hline \multicolumn{8}{|c|}{ Median values of RRMSE } \\
\hline GWEBLUP & 0.81 & 1.06 & 1.05 & 1.53 & 1.40 & 1.34 & 2.41 \\
\hline RGWEBLUP & 0.86 & 0.93 & 0.91 & 1.34 & 1.09 & .08 & 2.12 \\
\hline RGWEBLUP-bc & 0.80 & 0.93 & 0.92 & 1.04 & 1.16 & .15 & 1.67 \\
\hline EBLUP & 1.29 & 1.53 & 1.46 & 2.27 & 2.02 & 1.99 & 2.52 \\
\hline REB & 1.25 & 1.32 & 1.27 & 2.24 & 1.53 & .47 & 2.42 \\
\hline REBLUP-bc & 1.25 & 1.36 & 1.30 & 2.13 & 1.55 & 1.52 & 2.22 \\
\hline \multicolumn{8}{|c|}{ Spatial stationarity } \\
\hline \multicolumn{8}{|c|}{ Median values of $R B$} \\
\hline GWEBLUP & 0.01 & 0.01 & 0.01 & 0.07 & 0.08 & 0.11 & -1.78 \\
\hline RGWEBLUP & 0.00 & 0.02 & 0.01 & 0.07 & -0.55 & -0.54 & -1.59 \\
\hline RGWEBLUP-bc & -0.01 & 0.03 & 0.02 & 0.04 & -0.59 & -0.58 & -1.03 \\
\hline EBLUP & 0.00 & 0.02 & 0.02 & 0.05 & 0.26 & 0.27 & -2.08 \\
\hline REBLUP & -0.00 & 0.02 & 0.02 & 0.04 & -0.50 & -0.50 & -1.32 \\
\hline REBLUP-bc & -0.01 & 0.02 & 0.02 & 0.03 & -0.57 & -0.57 & -0.62 \\
\hline \multicolumn{8}{|c|}{ Median values of RRMSE } \\
\hline GWEBLUP & 0.84 & 1.10 & 1.10 & 1.80 & 1.49 & 1.48 & 2.82 \\
\hline RGWEBLUP & 0.86 & 0.93 & 0.92 & 1.35 & 1.20 & 1.19 & 2.10 \\
\hline RGWEBLUP-bc & 0.90 & 1.04 & 1.04 & 1.13 & 1.31 & 1.31 & 1.58 \\
\hline EBLUP & 0.80 & 1.07 & 1.06 & 1.67 & 1.56 & 1.55 & 2.80 \\
\hline REBLUP & 0.81 & 0.90 & 0.90 & 1.24 & 1.12 & 1.11 & 1.69 \\
\hline REBLUP-bc & 0.90 & 1.04 & 1.04 & 1.08 & 1.28 & 1.28 & 1.24 \\
\hline
\end{tabular}

and RGWEBLUP) suffer from bias in the stationary and non-stationary scenarios as a consequence of their assumption that the outliers have zero expectations (Chambers et al., 2014). In contrast, the robust predictive approaches (REBLUP-bc and RGWEBLUP-bc) correct for the bias especially in the areas 3740. Turning to the RRMSE results, we observe that the robust geographically weighted methods (RGWEBLUP and RGWEBLUP-bc) offer better performance compared with the non-robust GWEBLUP. These results indicate the benefit of combining geographically weighted regression methods with robust protection, offered by robust predictive or projective estimators, can lead to the gains in efficiency.

We now assess the performance of the proposed MSE estimators. Our main interest is in the performance of the MSE estimators for the geographically weighted regression methods (GWEBLUP, RGWEBLUP and RGWEBLUP-bc). In particular, we investigate the scenarios $(0,0),(v, e)_{s}$ and $(v, e)_{n s}$ under spatial non-stationarity in detail. However, we also present the results of the MSE estimators when used 
Table 2: Simulation results of RMSE estimators for spatial non-stationary population model

\begin{tabular}{|c|c|c|c|c|c|c|c|c|}
\hline \multirow[t]{2}{*}{ Predictor } & \multirow{2}{*}{$\begin{array}{c}\text { MSE } \\
\text { estimator }\end{array}$} & \multicolumn{7}{|c|}{ Results $(\%)$ for the following scenarios and areas } \\
\hline & & $\begin{array}{r}(0,0) \\
1-40\end{array}$ & $\begin{array}{r}(v, e)_{s} \\
1-40\end{array}$ & $\begin{array}{r}(v, e)_{s} \\
1-36\end{array}$ & $\begin{array}{c}(v, e)_{s} \\
37-40\end{array}$ & $\begin{array}{r}(v, e)_{n s} \\
1-40\end{array}$ & $\begin{array}{r}(v, e)_{n s} \\
1-36\end{array}$ & $\begin{array}{r}(v, e)_{n s} \\
37-40\end{array}$ \\
\hline \multicolumn{9}{|c|}{ Median value of $R B$} \\
\hline \multirow[t]{2}{*}{ GWEBLUP } & CCST & 3.73 & 5.00 & 6.29 & -16.93 & 13.60 & 14.54 & -19.75 \\
\hline & CCT & -23.05 & -17.64 & -17.30 & -29.21 & -7.22 & -7.10 & -27.58 \\
\hline \multirow[t]{2}{*}{ RGWEBLUP } & CCST & -5.81 & 4.34 & 4.70 & -22.18 & 9.16 & 10.64 & -37.37 \\
\hline & $\mathrm{CCT}$ & -25.57 & -11.06 & -10.38 & -30.35 & 1.50 & 4.40 & -38.40 \\
\hline \multirow{2}{*}{ RGWEBLUP-bc } & CCST & -4.06 & -5.06 & -4.10 & -17.84 & -11.01 & -10.33 & -43.03 \\
\hline & $\mathrm{CCT}$ & 1.65 & 5.63 & 6.43 & -9.53 & 6.30 & 7.51 & -31.32 \\
\hline \multicolumn{9}{|c|}{ Median value of RRMSE } \\
\hline \multirow[t]{2}{*}{ GWEBLUP } & CCST & 30.24 & 37.46 & 36.04 & 38.78 & 46.96 & 47.63 & 44.30 \\
\hline & CCT & 39.71 & 49.68 & 49.41 & 49.98 & 58.62 & 59.58 & 53.30 \\
\hline \multirow[t]{2}{*}{ RGWEBLUP } & CCST & 32.80 & 42.20 & 42.68 & 39.82 & 56.43 & 56.79 & 50.88 \\
\hline & $\mathrm{CCT}$ & 44.89 & 55.21 & 56.21 & 51.55 & 72.76 & 73.39 & 57.42 \\
\hline \multirow[t]{2}{*}{ RGWEBLUP-bc } & CCST & 31.97 & 34.84 & 34.89 & 33.31 & 36.63 & 36.53 & 47.24 \\
\hline & CCT & 39.34 & 51.63 & 52.37 & 45.01 & 64.87 & 66.00 & 49.75 \\
\hline
\end{tabular}

for estimating the MSE of the global estimators (EBLUP, REBLUP and REBLUP-bc) for these scenarios as well as the results for the spatial stationary scenarios in the supporting information.

MSE estimation for the RGWEBLUP is implemented by the pseudo-linearization MSE estimator (18) and by the linearization-based MSE estimator (30). Following Chambers et al. (2014), we refer to the pseudo-linearization and to the linearization-based MSE estimators as CCT and CCST MSE estimators respectively. For RGWEBLUP-bc the CCT (21) and the CCST (32) MSE estimators are used. The MSE estimator for the global estimators (EBLUP, REBLUP and REBLUP-bc) and for the GWEBLUP are also implemented by the CCT and CCST.

Table 2 reports the performance of the MSE estimators for the spatial non-stationary scenarios and presents the median values of the area-specific RB and RRMSE. The empirical MSE (over Monte-Carlo replications) is treated as the true MSE. As a general comment, we notice that the CCST offers better stability (in terms of lower RRMSE) compared to the CCT for the geographically weighted methods (GWEBLUP, RGWEBLUP and RGWEBLUP-bc) for all scenarios. In particular, first we notice that the CCST MSE estimator performs consistently better (in terms of bias and stability) compared to the CCT MSE estimator for all scenarios for the non-robust GWEBLUP. The CCT MSE estimator for the GWEBLUP underestimates the true MSE in all scenarios because the variability caused by the estimation of the variance components is not captured. Second, we focus on the performance of the MSE estimators for the robust projective RGWEBLUP. As already mentioned, the CCST MSE estimator is superior in terms of stability compared to the CCT MSE estimator. In terms of bias the picture is not as clear. In particular, the CCST MSE estimator shows a smaller bias for $(0,0)$ and for symmetric outlier contamination $(v, e)_{s}$, but the CCT performs better for asymmetric outlier contamination $(v, e)_{n s}$ especially in areas 1-36. However, the bias for both MSE estimators is of the same order of magnitude for scenarios 
with asymmetric outlier contamination especially in areas 37-40. Third, we concentrate on the MSE estimators for the robust predictive RGWEBLUP-bc. Similar to the RGWEBLUP, the CCST MSE estimator for the RGWEBLUP-bc offers more stability compared to the CCT, whereas the bias for both MSE estimators is of the same order of magnitude. These results indicate that using the CCST MSE estimator for the geographically weighted methods (GWEBLUP, RGWEBLUP and RGWEBLUP-bc) appears to have appealing properties regarding bias and stability in these scenarios.

\section{Application: Small area estimation of average total cash costs for farms in Australia}

We investigated in Section 4 the performance of the proposed methodology under known outlier contamination mechanism. However, in the context of real data the contamination mechanism is unknown. In this section we apply the proposed small area methods for estimating average total cash costs for regions in Australia.

The data of interest for this application comes from the Australian Agricultural and Gazing Industries Survey (AAGIS) conducted annually for over 30 years by the Australian Bureau of Agricultural and Resource Economics and Sciences (ABARES). The survey design is a single stage stratified random sampling with strata defined by ABARES regions, industry domains and size of the farms. The survey is designed from a population list from the Australian Business Register and provides a wide range of information on the economic performance of farm business units in Australia. For further information we refer to ABARES (2011). The small areas of interest are the ABARES regions which divide the Country by three dimensions - State, Zones (pastoral zone, wheat-sheep zone, high rainfall zone) and dairy regions. In total there are 32 ABARES regions in Australia. The sample we had access to is from 1991, where 951 farms from 12 out of the 32 regions are sampled. In addition to the survey, small area estimation is supported by additional auxiliary information from the Australian Business Register. However, the Australian Business Register contains highly sensitive information of farms and is governed by strict confidentiality rules. According to Chandra et al. (2012) we generated in the application a synthetic register for 12 out of the 32 regions by resampling with replacement from the AAGIS survey using selection probabilities inversely proportional to the sample weights.

In this application we want to provide reliable small area estimates of the average total cash costs for farms for the ABARES regions in Australia. The variable of interest is total cash costs in thousand Dollar which involves all payments made by the farm business for materials, services and labor. In addition we have access to the following potential covariates: (1) land area which includes all land operated by the farm business, (2) stock-beef which consists of the total number of closing stock-beef by the farm business, (3) stock-sheep, (4) harvested wheat which consists of the total quantity of harvested wheat by the farm business. Table 3 shows the distribution of these variables. The unconditional distribution of total cash costs indicates clear asymmetry. Therefore we analyse the conditional distribution of total cash costs given the available covariates. 
Table 3: Summary statistics for the continuous variables in the model

\begin{tabular}{lrrrrrr}
\hline & Min. & 1st Qu. & Median & Mean & 3rd Qu. & Max. \\
\hline Total cash costs & 5.30 & 76.90 & 146.50 & 283.80 & 257.90 & 31910.00 \\
Land area & 14.10 & 647.00 & 1392.00 & 3287.00 & 2944.00 & 122000.00 \\
Stock-beef & 0.00 & 0.00 & 29.50 & 319.40 & 254.50 & 23390.00 \\
Stock-sheep & 0.00 & 141.80 & 1453.00 & 3033.00 & 3754.00 & 222000.00 \\
Harvested wheat & 0.00 & 0.00 & 155.00 & 537.70 & 612.50 & 11500.00 \\
\hline
\end{tabular}

We use a 2-level random intercepts model (level $1=$ farm; level $2=$ region) for the variable of interest with random effects specified at the level of regions. The covariates in the model are land area, stock-beef, stock-sheep, harvested wheat and four indicator variables to control for different industries (specialist crop farms, mixed livestock and crop farms, sheep specialists, beef specialists, mixed sheep and beef farms). This model serves as a first starting point.

Evidence of spatial non-stationarity of the regression coefficients in the data is also present. We conducted a likelihood ratio (LR) test suggested by Fotheringham et al. (2002) where the likelihood of the global (stationary) model (1) is compared to the likelihood of the local (non-stationary) model (3). According to the p-value of the LR-Test $\left(<1.4 e^{-213}\right)$ the assumption of stationarity of the regression coefficients was rejected. It seems that an approach based on geographical weighted regression is appropriate in this application.

The non-stationary model (3) is based on distributional assumptions. To assess departures from these assumptions, Figure 5 shows normal probability plots of level 1 and level 2 residuals. In both levels we can observe severe departures from the Gaussian assumptions, which may be caused by outliers in the sample. Applying a robust estimation method to control for the influence of the extreme observations may be recommendable in this application.

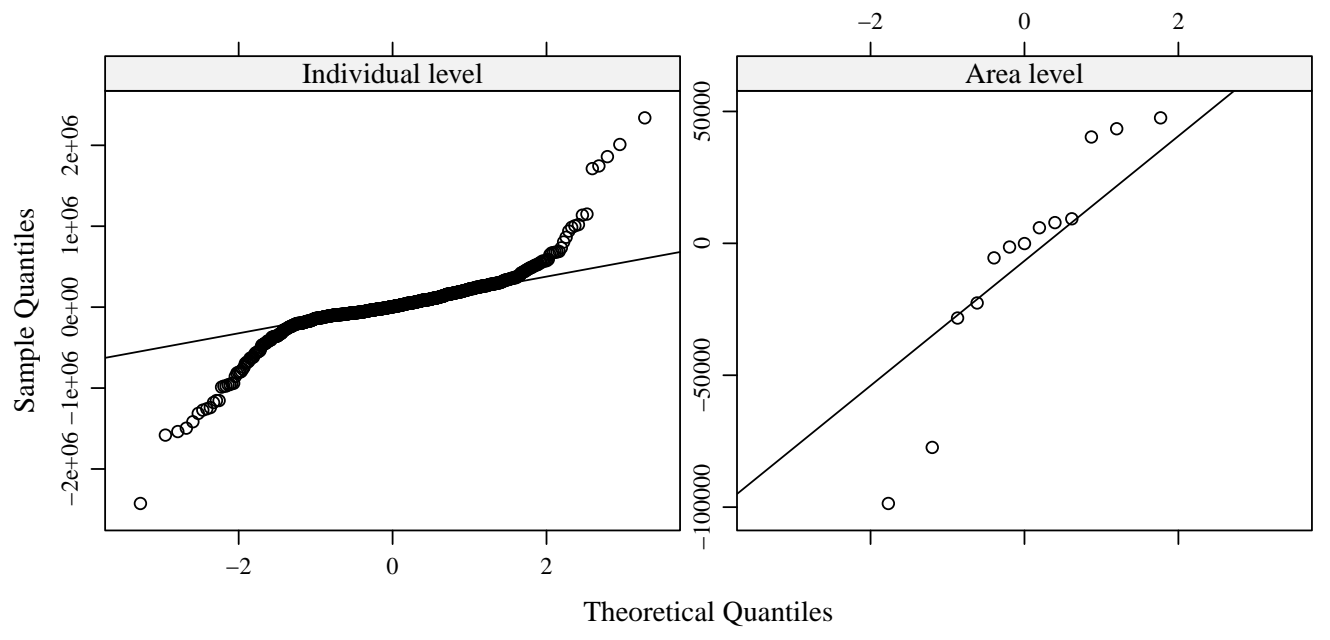

Figure 1: Normal probability plots of level 1 (left) and level 2 (right) residuals.

Based on the diagnostics we decided to produce estimates of average total cash costs for each region by the GWEBLUP (4), the robust projective estimator RGWEBLUP (10) and the robust predictive esti- 
mator RGWEBLUP-bc (13). For comparison reasons we also incorporated the direct estimator (Horvitz and Thompson, 1952). Note that direct estimation is not an option for the AAGIS survey because around $60 \%$ of the regions are out-of-sample.

Table 4: Average total cash costs of regions (in thousand Dollars)

\begin{tabular}{cccccc}
\hline \multirow{2}{*}{$\begin{array}{c}\text { Region } \\
\text { Sample } \\
\text { size }\end{array}$} & \multicolumn{4}{c}{ Results for the following estimators } \\
\cline { 3 - 6 } & & GWEBLUP & RGWEBLUP & RGWEBLUP-bc & Direct \\
\hline 121 & 95 & 138.39 & 173.68 & 178.95 & 192.01 \\
122 & 103 & 18.38 & 107.58 & 103.03 & 107.21 \\
123 & 108 & 69.40 & 135.39 & 137.57 & 130.88 \\
221 & 56 & 118.40 & 108.29 & 114.74 & 102.17 \\
222 & 60 & 80.73 & 88.95 & 91.85 & 86.06 \\
223 & 72 & 56.90 & 71.85 & 76.31 & 74.67 \\
321 & 79 & 61.54 & 85.15 & 90.59 & 92.68 \\
322 & 117 & 121.67 & 163.84 & 160.33 & 162.78 \\
421 & 51 & 146.66 & 123.29 & 117.14 & 118.02 \\
422 & 80 & 135.20 & 103.22 & 105.23 & 97.34 \\
521 & 83 & 179.90 & 180.79 & 186.08 & 180.36 \\
522 & 47 & 220.18 & 203.61 & 197.53 & 212.40 \\
\hline
\end{tabular}

Table 4 presents the estimated average total cash costs for regions in Australia. We first observe that there are differences between the non-robust (GWEBLUP) and the robust estimates (RGWEBLUP and RGWEBLUP-bc) for some regions (i.e. region 122, 123, 223, 322 and 422). In order to judge the quality of the model-based estimators, we assess the following criteria (Chambers et al., 2016): high proximity to the direct estimates. Following Brown et al. (2001), we investigate the criteria by computing a goodness-of-fit test. The idea of the test is that model-based estimators should not differ significantly from unbiased direct estimators. The test is computed as the value of the Wald statistic

$$
W\left(\hat{\bar{y}}_{i}^{\text {model }}\right)=\sum_{i=1}^{m} \frac{\left(\hat{\bar{y}}_{i}^{\text {direct }}-\hat{\bar{y}}_{i}^{\text {model }}\right)^{2}}{\widehat{\operatorname{Var}}\left(\hat{\bar{y}}_{i}^{\text {direct }}\right)+\widehat{M S E}\left(\hat{\bar{y}}_{i}^{\text {model }}\right)},
$$

where $\hat{\bar{y}}_{i}^{\text {model }}$ is a generic notation used to designate a model-based estimator of the small area average introduced in Section 2. The MSE of $\hat{\bar{y}}_{i}^{\text {model }}$ is calculated by using the proposed CCT (Section 3.1) and CCST (Section 3.2) approaches. Under the null hypothesis the test statistic $W$ is $\chi^{2}$-distributed with $m$ degrees of freedom where $m$ denotes the number of sampled areas. The results of the test are

Table 5: Results for Goodness-of-fit test

\begin{tabular}{|c|c|c|c|c|c|c|}
\hline \multirow{2}{*}{$\begin{array}{l}\text { MSE estimator } \\
\text { Estimator }\end{array}$} & \multicolumn{3}{|c|}{$C C S T$} & \multicolumn{3}{|c|}{$C C T$} \\
\hline & $W$ & $D F$ & $p$-value & $W$ & $D F$ & $p$-value \\
\hline GWEBLUP & 369.51 & 12 & 0.00 & 442.09 & 12 & 0.00 \\
\hline RGWEBLUP & 12.14 & 12 & 0.43 & 11.66 & 12 & 0.47 \\
\hline RGWEBLUP-bc & 18.79 & 12 & 0.09 & 17.60 & 12 & 0.13 \\
\hline
\end{tabular}


reported in Table 5. We note that the robust estimates (RGWEBLUP and RGWEBLUP-bc) are not statistically different from the direct estimators. In contrast, the small area estimates of the non-robust GWEBLUP differ significantly from the direct estimates. In addition, we note that the RGWEBLUP and RGWEBLUP-bc seem to be consistent with the direct estimates, with a correlation of 0.989 and 0.988 respectively, whereas the correlation between the GWEBLUP and the direct estimates is 0.739 . These results provide additional evidence that using a robust estimator (RGWEBLUP or RGWEBLUP-bc) is appropriate in this application.

Table 6: Results for MSE estimates

\begin{tabular}{lccccc}
\hline \multirow{2}{*}{ MSE estimator } & \multicolumn{3}{c}{ CCST } & & \multicolumn{2}{c}{$C C T$} \\
\cline { 2 - 3 } \cline { 5 - 6 } Region & 29.74 & 18.28 & & 34.02 & 27.46 \\
\hline 121 & 29.89 & 13.69 & & 30.12 & 15.37 \\
122 & 24.66 & 13.06 & & 24.60 & 14.07 \\
123 & 26.72 & 21.24 & & 27.17 & 22.07 \\
221 & 30.55 & 18.63 & & 30.43 & 19.14 \\
222 & 71.84 & 20.61 & & 79.00 & 46.39 \\
223 & 26.70 & 19.73 & & 28.60 & 22.55 \\
321 & 29.53 & 15.46 & & 33.12 & 22.80 \\
322 & 27.44 & 23.49 & & 26.97 & 23.90 \\
421 & 16.09 & 14.77 & & 16.32 & 16.02 \\
422 & 19.97 & 15.12 & & 19.79 & 18.38 \\
521 & 23.02 & 23.17 & & 22.41 & 24.81 \\
522 & 29.68 & 18.11 & & 31.05 & 22.75 \\
\hline Mean & 27.08 & 18.46 & & 27.89 & 22.31 \\
Median & & & & &
\end{tabular}

MSE estimates for the robust estimators, using the two analytic approaches (CCT and CCST) described in Section 3.1 and 3.2, are presented in Table 6. We note that the estimated MSE of the RGWEBLUP-bc are generally lower than those of the RGWEBLUP. This indicates potentially better accuracy obtained by applying a bias-corrected small area estimator. Based on the point and MSE estimates, we can conclude that the RGWEBLUP-bc appears to be a good choice for producing small area estimates of average total cash costs for ABARES regions in Australia.

\section{Concluding remarks}

We propose outlier robust projective (RGWEBLUP) and predictive (RGWEBLUP-bc) extensions to the currently available geographically weighted EBLUP. The advantages of the introduced methods are twofold: first, the robust nature of the estimators reduce the influence of outliers and protect against departures of the underlying model assumption; second, the proposed methods accounts for the presence of spatial non-stationarity in the data. MSE estimation is conducted by two analytic MSE estimators (CCT and CCST) that account for the spatial dependence. The results from our model-based simulation indicate the potential benefit of combining geographically weighted regression with robust estimation. In addition, the proposed CCST MSE estimator for the RGWEBLUP and RGWEBLUP-bc appears to have 
appealing properties in terms of bias and stability in our scenarios. We demonstrate in the application that the robust geographically weighted EBLUPs are of relevance and appear to be a good choice for producing regionally disaggregated estimates of total cash costs of farms in Australia.

There are some outstanding research issues. First, the choice of the tuning constant is a challenging problem in robust estimation. We rely in this paper on a a-priori choice of the tuning constant. The use of data-driven tuning constants for prediction may be an avenue for further research. One idea is to use the ideas of Y. Wang (2007) who chooses the tuning constant by minimizing the asymptotic variance of the regression coefficients. However, this approach is not necessarily optimal in terms of prediction. Second, another avenue for further research is to investigate alternative ways for correcting the bias of the robust projective estimator (RGWEBLUP) under spatial non-stationarity. Dongmo-Jiongo et al. (2013) introduced a global-bias correction which is based on information from other areas. It would be interesting to extend this approach to account for spatial non-stationarity in the data. Third, developing bootstrap MSE estimators under spatial non-stationarity similar to Chambers and Chandra (2013) offers another avenue for further research.

\section{Acknowledgements}

The research leading to these results has received support under the European Commissions's 7th Framework Programme (FP7/2013-2017) under grant agreement no312691, InGRID - Inclusive Growth Research Infrastructure Diffusion. The work of Salvati has been developed under the support of the project PRIN-SURWEY http://www. sp.unipg.it/surwey/ (Grant 2012F42NS8, Italy).

\section{Appendix A}

In order to develop a Fix-Point algorithm for the approximation of $\boldsymbol{\beta}_{\left(u_{k}\right)}$ we need to find an expression of the form $\boldsymbol{\beta}_{\left(u_{k}\right)}=f\left(\boldsymbol{\beta}_{\left(u_{k}\right)}\right)$ where $f$ is an arbitrary continuous function. Therefor we rewrite expression (6) as

$$
\alpha\left(\boldsymbol{\beta}_{\left(u_{k}\right)}\right)=\boldsymbol{X}^{T} \boldsymbol{V}_{k}^{-1} \boldsymbol{U}_{k}^{1 / 2} \boldsymbol{D}_{k}\left(\boldsymbol{\beta}_{\left(u_{k}\right)}\right) \boldsymbol{U}_{k}^{-1 / 2}\left(\boldsymbol{y}-\boldsymbol{X} \boldsymbol{\beta}_{\left(u_{k}\right)}\right)=\mathbf{0}
$$

with $\boldsymbol{D}_{k}\left(\boldsymbol{\beta}_{\left(u_{k}\right)}\right)=\psi\left\{\boldsymbol{U}_{k}^{-1 / 2}\left(\boldsymbol{y}-\boldsymbol{X} \boldsymbol{\beta}_{\left(u_{k}\right)}\right) / \boldsymbol{U}_{k}^{-1 / 2}\left(\boldsymbol{y}-\boldsymbol{X} \boldsymbol{\beta}_{\left(u_{k}\right)}\right)\right.$. It follows directly that

$$
\boldsymbol{\beta}_{\left(u_{k}\right)}=\left(\boldsymbol{X}^{T} V_{k}^{-1} \boldsymbol{U}_{k}^{1 / 2} \boldsymbol{D}_{k}\left(\boldsymbol{\beta}_{\left(u_{k}\right)}\right) \boldsymbol{U}_{k}^{-1 / 2} \boldsymbol{X}\right)^{-1} \boldsymbol{X}^{T} \boldsymbol{V}_{k}^{-1} \boldsymbol{U}_{k}^{1 / 2} \boldsymbol{D}_{k}\left(\boldsymbol{\beta}_{\left(u_{k}\right)}\right) \boldsymbol{U}_{k}^{-1 / 2} \boldsymbol{y}
$$

and therefor $\boldsymbol{\beta}_{\left(u_{k}\right)}$ can be approximated by repeatedly evaluating expression

$$
\boldsymbol{\beta}_{\left(u_{k}\right)}^{m}=\left(\boldsymbol{X}^{T} \boldsymbol{V}_{k}^{-1} \boldsymbol{U}_{k}^{1 / 2} \boldsymbol{D}_{k}\left(\boldsymbol{\beta}_{\left(u_{k}\right)}^{m-1}\right) \boldsymbol{U}_{k}^{-1 / 2} \boldsymbol{X}\right)^{-1} \boldsymbol{X}^{T} \boldsymbol{V}_{k}^{-1} \boldsymbol{U}_{k}^{1 / 2} \boldsymbol{D}_{k}\left(\boldsymbol{\beta}_{\left(u_{k}\right)}^{m-1}\right) \boldsymbol{U}_{k}^{-1 / 2} \boldsymbol{y}
$$

until convergence is reached. 


\section{Appendix B}

The steps of the algorithm for the estimation of $\boldsymbol{\beta}_{\left(u_{i j}\right)}, \boldsymbol{\theta}$ and $\boldsymbol{v}$ are as follows:

1. Compute all in-sample distances to obtain the matrix $W_{k}$ for each sample location $k \in s$.

2. Estimate the optimal bandwidth $b$ by using cross validation (see Chandra et al. (2012) for details).

3. Choose initial values $\boldsymbol{B}_{s}^{(0)}$ and $\boldsymbol{\theta}^{(0)}$.

4. Update $\boldsymbol{B}_{s}^{(m+1)}$ according to the Fix-Point algorithm.

5. Estimate the residuals $\boldsymbol{r}=\boldsymbol{y}-\left(\boldsymbol{X} \circ \boldsymbol{B}_{s}^{(m+1)}\right) \mathbf{1}_{p}$ and update $\boldsymbol{\theta}^{(m+1)}$ according to the Fix-Point algorithm.

6. Return to step 4 and continue until convergence is achieved.

7. Define the estimates at convergence to be the robust ML-estimators $\hat{\boldsymbol{B}}_{s}^{\psi, g w}$ and $\hat{\boldsymbol{\theta}}^{\psi, g w}$.

8. Plug $\hat{\boldsymbol{B}}_{s}^{\psi, g w}$ and $\hat{\boldsymbol{\theta}}^{\psi, g w}$ into (9) and estimate $\boldsymbol{v}$ by using the Fisher scoring algorithm.

9. Define the estimates at convergence to be the robust ML-estimator $\hat{\boldsymbol{v}}^{\psi, g w}$.

10. Compute all out-of-sample distances to obtain the matrix $W_{k}$ for each non-sampled location $k \in r$.

11. Compute $\hat{\boldsymbol{V}}_{i j}=\boldsymbol{Z} \hat{\boldsymbol{\Sigma}}_{v} \boldsymbol{Z}^{T}+\hat{\boldsymbol{\Sigma}}_{e} \boldsymbol{W}_{i j}^{-1}$ for each non-sampled unit.

12. Estimate $\boldsymbol{\beta}_{\left(u_{i j}\right)}$ for the non-sampled units by $\hat{\boldsymbol{\beta}}_{\left(u_{i j}\right)}^{\psi, g w}=\left(\boldsymbol{X}^{T} \hat{\boldsymbol{V}}_{i j}^{-1} \boldsymbol{X}\right)^{-1} \boldsymbol{X}^{T} \hat{\boldsymbol{V}}_{i j}^{-1} \boldsymbol{y}^{*}$.

Note that we have a closed form solution for $\boldsymbol{\beta}_{\left(u_{i j}\right)}$ for the non-sampled units because we interpret that $\boldsymbol{y}^{*}$ as pseudo-observations without outlier contamination and we assume that model (3) holds. The values $\boldsymbol{y}^{*}$ from step 12 are defined by equation (16).

\section{Appendix C}

We can rewrite the ML estimating equation (9) as

$$
\Delta(\boldsymbol{v})=\boldsymbol{Z}^{T} \boldsymbol{\Sigma}_{e}^{-1 / 2} \boldsymbol{D}_{2} \boldsymbol{\Sigma}_{e}^{-1 / 2}\left(\boldsymbol{y}-\boldsymbol{\rho}_{s}-\boldsymbol{Z} \boldsymbol{v}\right)-\boldsymbol{\Sigma}_{v}^{-1 / 2} \boldsymbol{D}_{3} \boldsymbol{\Sigma}_{v}^{-1 / 2} \boldsymbol{v}=\mathbf{0}
$$

with $\boldsymbol{D}_{2}$ and $\boldsymbol{D}_{3}$ being defined as in expression (15). It follows that

$$
\begin{aligned}
\boldsymbol{v} & =\underbrace{\left(\boldsymbol{Z}^{T} \boldsymbol{\Sigma}_{e}^{-1 / 2} \boldsymbol{D}_{2} \boldsymbol{\Sigma}_{e}^{-1 / 2} \boldsymbol{Z}+\boldsymbol{\Sigma}_{v}^{-1 / 2} \boldsymbol{D}_{3} \boldsymbol{\Sigma}_{v}^{-1 / 2}\right)^{-1} \boldsymbol{Z}^{T} \boldsymbol{\Sigma}_{e}^{-1 / 2} \boldsymbol{D}_{2} \boldsymbol{\Sigma}_{e}^{-1 / 2}}_{=\boldsymbol{Q}}\left(\boldsymbol{y}-\boldsymbol{\rho}_{s}\right) \\
& =\boldsymbol{Q}\left(\boldsymbol{I}_{n}-\boldsymbol{H}\right) \boldsymbol{y}
\end{aligned}
$$

with $\boldsymbol{H}$ being defined as in expression (15). Further, for the non-sampled units we can rewrite the regression coefficients $\boldsymbol{\beta}_{\left(u_{k}\right)}$ as

$$
\boldsymbol{\beta}_{\left(u_{k}\right)}=\left(\boldsymbol{X}^{T} \boldsymbol{V}_{k}^{-1} \boldsymbol{X}\right)^{-1} \boldsymbol{X}^{T} \boldsymbol{V}_{k}^{-1} \boldsymbol{D}_{1} \boldsymbol{y}
$$


with $\boldsymbol{D}_{1}$ being defined as in (15). Plugging expressions (36) and (37) into (10) yields

$$
\begin{aligned}
\hat{\bar{y}}_{i}^{R G W E B L U P} & =N_{i}^{-1}\left\{\sum_{j \in s_{i}} y_{i j}+\sum_{j \in r_{i}}[\underbrace{\boldsymbol{x}_{i j}^{T}\left(\boldsymbol{X}^{T} \hat{\boldsymbol{V}}_{i j}^{-1} \boldsymbol{X}\right)^{-1} \boldsymbol{X}^{T} \hat{\boldsymbol{V}}_{i j}^{-1} \boldsymbol{D}_{1}}_{=\boldsymbol{\nu}_{i j}} \boldsymbol{y}+\underbrace{\boldsymbol{z}_{i}^{T} \boldsymbol{Q}\left(\boldsymbol{I}_{n}-\boldsymbol{H}\right)}_{=\boldsymbol{\xi}_{i j}} \boldsymbol{y}]\right\} \\
& =\underbrace{N_{i}^{-1}\left[\boldsymbol{\delta}_{i}^{T}+\left(N_{i}-n_{i}\right)\left(\overline{\boldsymbol{\nu}}_{i}+\overline{\boldsymbol{\xi}}_{i}\right)\right]}_{=\left(\boldsymbol{d}_{i}^{R G W E B L U P}\right)^{T}} \boldsymbol{y} .
\end{aligned}
$$

\section{Appendix D}

We can rewrite the ML estimating equation (6) as

$$
\alpha\left(\boldsymbol{\beta}_{\left(u_{k}\right)}\right)=\boldsymbol{X}^{T} \boldsymbol{V}_{k}^{-1} \boldsymbol{U}_{k}^{1 / 2} \boldsymbol{D}_{4(k)} \boldsymbol{U}_{k}^{-1 / 2}\left(\boldsymbol{y}-\boldsymbol{X} \boldsymbol{\beta}_{\left(u_{k}\right)}\right)=\mathbf{0}
$$

with $\boldsymbol{D}_{4(k)}$ being defined as in (15). It follows that for the sampled units we can rewrite the regression coefficients $\boldsymbol{\beta}_{\left(u_{k}\right)}$ as

$$
\boldsymbol{\beta}_{\left(u_{k}\right)}=\left(\boldsymbol{X}^{T} V_{k}^{-1} \boldsymbol{U}_{k}^{1 / 2} \boldsymbol{D}_{4(k)} \boldsymbol{U}_{k}^{-1 / 2} \boldsymbol{X}\right)^{-1} \boldsymbol{X}^{T} \boldsymbol{V}_{k}^{-1} \boldsymbol{U}_{k}^{1 / 2} \boldsymbol{D}_{4(k)} \boldsymbol{U}_{k}^{-1 / 2} \boldsymbol{y} .
$$

Using the upper expression and the results from Appendix $\mathrm{C}$ we can rewrite the bias correction in expression (13) as

$$
\begin{aligned}
b c_{i}^{g w} & =\left(\frac{N_{i}-n_{i}}{N_{i}}\right) \frac{1}{\sum_{j \in s_{i}} \bar{w}_{i j}} \sum_{j \in s_{i}} q_{i j}\left(y_{i j}-\mathbf{x}_{i j} \hat{\boldsymbol{\beta}}_{\left(u_{i j}\right)}^{\psi, g w}-\hat{v}_{i}^{\psi, g w}\right) \\
& =\left(\frac{N_{i}-n_{i}}{N_{i}}\right) \frac{1}{\sum_{j \in s_{i}} \bar{w}_{i j}} \sum_{j \in s_{i}} q_{i j}\left(y_{i j}-\boldsymbol{h}_{i j} \boldsymbol{y}-\boldsymbol{\xi}_{i j} \boldsymbol{y}\right)
\end{aligned}
$$

with $q_{i j}$ being defined as in expression (23) and $\boldsymbol{h}_{i j}$ as in expression (15). Plugging the upper expression into (13) and using the results from Appendix $\mathrm{C}$ it follows that 


$$
\begin{aligned}
\hat{\bar{y}}_{i}^{R G W E B L U P-b c}= & \hat{\bar{y}}_{i}^{R G W E B L U P}+b c_{i}^{g w} \\
= & N_{i}^{-1}\left[\boldsymbol{\delta}_{i}^{T}+\left(N_{i}-n_{i}\right)\left(\overline{\boldsymbol{\nu}}_{i}+\overline{\boldsymbol{\xi}}_{i}\right)\right] \boldsymbol{y}+\left(\frac{N_{i}-n_{i}}{N_{i}}\right) \frac{1}{\sum_{j \in s_{i}} \bar{w}_{i j}} \sum_{j \in s_{i}} q_{i j}\left(y_{i j}-\boldsymbol{h}_{i j} \boldsymbol{y}-\boldsymbol{\xi}_{i j} \boldsymbol{y}\right) \\
= & N_{1}^{-1}\left\{\left(\boldsymbol{1}_{n}+\frac{N_{i}-n_{i}}{\sum_{j \in s_{i}} \bar{w}_{i j}} \boldsymbol{q}\right)^{T} \circ \boldsymbol{\delta}_{i}^{T}\right. \\
& +\left(N_{i}-n_{i}\right)\left(\overline{\boldsymbol{\nu}}_{i}-\frac{1}{\sum_{j \in s_{i}} \bar{w}_{i j}} \sum_{j \in s_{i}} q_{i j} \boldsymbol{h}_{i j}\right) \\
& \left.+\left(N_{i}-n_{i}\right)\left(\overline{\boldsymbol{\xi}}_{i}-\frac{1}{\sum_{j \in s_{i}} \bar{w}_{i j}} \sum_{j \in s_{i}} q_{i j} \boldsymbol{\xi}_{i j}\right)\right\} \boldsymbol{y} \\
= & \left(\boldsymbol{d}_{i}^{R G W E B L U P-b c}\right)^{T} \boldsymbol{y},
\end{aligned}
$$

with $\boldsymbol{q}$ being defined as in expression (23).

\section{References}

ABARES (2011). Survey methods and definitions. Technical report, Australian Bureau of Agriculture and Resource Economics an Sciences, Downloaded from http://www.daff.gov.au/sitecollectiondocuments/abares/surveys/survey-definitions-methods.pdf (7th November 2015).

Battese, G. E., R. M. Harter, and W. A. Fuller (1988). An error component model for prediction of county crop areas using survey and satellite data. Journal of the American Statistical Association 83 (401), 28-36.

Brown, G., R. Chambers, P. Heady, and D. Heasman (2001). Evaluation of small area estimation methods an application to unemployment estimates from the uk lfs. In Proceedings of Statistics Canada Symposium 2001: Achieving Data Quality in a Statistical Agency: A Methodological Perspective. Statistics Canada.

Brunsdon, C., A. S. Fotheringham, and M. Charlton (1996). Geographically weighted regression: a method for exploring spatial nonstationarity. Geographical Analysis 28, 281-298.

Chambers, R. (1986). Outlier robust finite population estimation. Journal of the American Statistical Association 81, 1063-1069.

Chambers, R. and H. Chandra (2013). A random effect block bootstrap for clustered data. Journal of Computational and Graphical Statistics 22, 452-470.

Chambers, R., H. Chandra, N. Salvati, and N. Tzavidis (2014). Outlier robust small area estimation. Journal of the Royal Statistical Society: Series B 76 (1), 47-69. 
Chambers, R., J. Chandra, and N. Tzavidis (2011). On bias-robust mean squared error estimation for pseudo-linear small area estimators. Survey Methodology 37 (2), 153-170.

Chambers, R., N. Salvati, and N. Tzavidis (2016). Semiparametric small area estimation for binary outcomes with application to unemployment estimation for local authorities in the UK. Journal of the Royal Statistical Society: Series A (Statistics in Society) 179, 453-479.

Chambers, R. and N. Tzavidis (2006). M-quantile models for small area estimation. Biometrika 93 (2), $255-268$.

Chandra, H., N. Salvati, R. Chambers, and N. Tzavidis (2012). Small area estimation under spatial nonstationarity. Computational Statistics and Data Analysis 56, 2875-2888.

Chandra, H., N. Salvati, and R. R. Chambers (2015). A spatially nonstationary fay-herriot model for small area estimation. Journal of Survey Statistics and Methodology, forthcoming.

Chatrchi, G. (2012). Robust estimation of variance components in small area estimation. Master's thesis, Carleton University.

D’Alo, M., L. D. Consiglio, S. Falorsi, M. G. Ranalli, and F. Solari (2012). Use of spatial information in small area models for unemployment rate estimation at sub-provincial areas in italy. Journal of the Indian Society of Agricultural Statistics 66 (1), 43-53.

Dongmo-Jiongo, V., D. Haziza, and P. Duchesne (2013). Controlling the bias of robust small area estimators. Biometrika 100, 843-858.

Fay, R. E. and R. A. Herriot (1979). Estimation of income for small places: An application of james-stein procedures to census data. Journal of the American Statistical Association 74 (366), 269-277.

Fellner, W. H. (1986). Robust estimation of variance components. Technometrics 28 (1), 51-60.

Fotheringham, A. S., C. Brunsdon, and M. Charlton (2002). Geographically Weighted Regression. West Sussex: Wiley.

Giusti, C., S. Marchetti, M. Pratesi, and N. Salvati (2012). Semiparametric fay-herriot model using penalized splines. Journal of the Indian Society of Agricultural Statistics 66 (1), 1-14.

Harville, D. A. (1977). Maximum likelihood approaches to variance component estimation and to related problems. Journal of the American Statistical Association 72 (358), 320-340.

Henderson, C. R. (1975). Best linear unbiased estimation and prediction under a selection model. Biometrics 31 (2), 423-447.

Horvitz, D. and D. Thompson (1952). A generalization of sampling without replacement from a finite universe. Journal of the American Statistical Association 47, 663-685. 
Huber, P. J. (1964). Robust estimation of a location parameter. The Annals of Mathematical Statistical 35, 73-101.

Jiang, J. and P. Lahiri (2006). Mixed model prediction and small area estimation. Test 15 (1), 1-96.

Longford, N. (2010). Small area estimation with spatial similarity. Computational Statistics \& Data Analysis 54 (1), 1151-1166.

Opsomer, J., G. Claeskens, M. Ranalli, G. Kauermann, and F. Breidt (2008). Nonparametric small area estimation using penalized spline regression. Journal of the Royal Statistical Society: Series B 70, 265-283.

Porter, A. T., S. H. Holan, C. K. Wikle, and N. Cressie (2014). Spatial fay-herriot models for small area estimation with functional covariates. Spatial Statistics 10, 27-42.

Pratesi, M. and N. Salvati (2008). Small area estimation: the eblup estimator based on spatially correlated random area effects. Statistical Methods \& Applications 17, 113-141.

Rao, J. N. K. (2003). Small Area Estimation. New York: Wiley.

Rao, J. N. K. and I. Molina (2015). Small Area Estimation. 2nd Edition. New York: Wiley.

Salvati, N., N. Tzavidis, M. Pratesi, and R. Chambers (2012). Small area estimation via m-quantile geographically weighted regression. Test 21 (1), 1-28.

Schmid, T. and R. Münnich (2014). Spatial robust small area estimation. Statistical Papers 55, 653-670.

Schmid, T., N. Tzavidis, R. Münnich, and R. Chambers (2016). Outlier robust small area estimation under spatial correlation. Scandinavian Journal of Statistics, forthcoming.

Singh, B. B., G. K. Shukla, and D. Kundu (2005). Spatio-temporal models in small area estimation. Statistics Canada 31 (2), 183-195.

Sinha, S. K. and J. N. K. Rao (2009). Robust small area estimation. The Canadian Journal of Statistics 37 (3), 381-399.

Sugasawa, S., Y. Kawakubo, and K. Ogasawara (2015). Geographically weighted empirical bayes estimation via natural exponential family. Working paper arXiv:1508.01641.

Y. Wang, X. Lin, M. Z. Z. B. (2007). Robust estimation using the huber funtion with a data-dependent tuning constant. Journal of Computational and Graphical Statistics 16 (2), 468-481. 